\title{
Inertial-subrange structures of isotropic incompressible magnetohydrodynamic turbulence in the Lagrangian renormalized approximation
}

\author{
Kyo Yoshida ${ }^{\text {a) }}$ and Toshihico Arimitsu \\ Graduate School of Pure and Applied Sciences, University of Tsukuba, \\ 1-1-1 Tennoudai, Tsukuba, Ibaraki 305-8571, Japan
}

(Received 23 August 2006; accepted 10 February 2007; published online 18 April 2007)

\begin{abstract}
The structures of isotropic incompressible magnetohydrodynamic (MHD) turbulence in the inertial subrange are studied within the Lagrangian renormalized approximation (LRA). It is confirmed that LRA derives the total energy spectrum which is consistent with the Iroshnikov-Kraichnan (IK) spectrum. The residual energy spectrum in LRA is found to obey $k^{-2}$ scaling law, where $k$ is the wave number. Given are the quantitative estimates of (i) the dimensionless constants in the total and residual energy spectra, (ii) contribution of triad interactions to the energy flux, and (iii) the eddy viscosity and the eddy magnetic diffusivity. A direct numerical simulation (DNS) of a forced quasi-isotropic incompressible MHD turbulence is performed to find that the obtained total energy spectrum is in good agreement with the one derived within LRA both in its scaling exponent and in the dimensionless constant. The residual energy spectrum obtained in the DNS agrees with that derived in LRA with respect to the scaling exponent and the sign of the dimensionless constant, which is negative, although the magnitude of the dimensionless constant is about four times larger. (c) 2007 American Institute of Physics. [DOI: 10.1063/1.2717687]
\end{abstract}

\section{INTRODUCTION}

Turbulence occurring in a conducting fluid interacting with a magnetic field is described appropriately within the framework of magnetohydrodynamics (MHD) (see, e.g., Ref. 1). For incompressible MHD turbulence, the structure of an energy spectrum within the inertial subrange has been discussed by many authors, however it is still an issue to be debated.

Iroshnikov $^{2}$ and Kraichnan ${ }^{3}$ (hereafter, IK) suggested phenomenologically that the energy spectrum in the inertial subrange of isotropic incompressible MHD turbulence should be $E^{u}(k)=E^{B}(k)=A \epsilon^{1 / 2} B_{0}^{1 / 2} k^{-3 / 2}$ (hereafter, the IK spectrum). Here, $E^{u}(k)$ and $E^{B}(k)$ are the kinetic and magnetic energy spectrum, $k$ is a wave number, $\epsilon$ is the total energy dissipation rate per unit mass, $B_{0}$ is the root mean square of magnetic field along an arbitrary axis, and $A$ is a dimensionless constant. Note that, for purely hydrodynamic turbulence, the energy spectrum in the inertial subrange is given by the well-known Kolmogorov spectrum, i.e., $E^{u}(k)$ $\propto \epsilon^{2 / 3} k^{-5 / 3}$, where a possible intermittency correction is neglected. Pouquet and others ${ }^{4}$ developed a theory for MHD turbulence by making use of the eddy-damped quasinormal Markovian (EDQNM) approximation in order to be consistent with the IK spectrum in the high-wave-number limit. Within the framework, they obtained the correction to the IK spectrum, that is, $E^{R}(k) \propto k^{-2}$ and $E^{R}(k)<0$, where $E^{R}(k)$ $=E^{u}(k)-E^{B}(k)$ is the residual energy spectrum. They also treated both cases with nonzero magnetic helicity $\int_{V} d \mathbf{x B} \cdot \mathbf{A}$ (Ref. 4) and with nonzero cross helicity $\int_{V} d \mathbf{x u} \cdot \mathbf{B}$ (Refs. 5

a)Electronic mail: yoshida@sakura.cc.tsukuba.ac.jp and 6), where $\mathbf{u}$ is the velocity field, $\mathbf{B}$ is the magnetic field, and $\mathbf{A}$ is the vector potential.

Some authors claim that IK phenomenology should be modified by taking into account the local anisotropy of the magnetic field. Goldreich and Sridhar ${ }^{7-9}$ proposed three phenomenological models for weak, intermediate, and strong turbulences. In each model, they obtained the energy spectrum density as a function of $k_{\perp}$ and $k_{\|}$, where $k_{\perp}\left(k_{\|}\right)$denotes the wave-vector component perpendicular (parallel) to the local mean magnetic field $\mathbf{b}_{0}$ (see Ref. 10 for a review). Galtier et al. ${ }^{11}$ claimed that, for globally isotropic weak turbulence, the local anisotropy yields $k^{-2}$ scaling for the total energy spectrum $E(k)=E^{u}(k)+E^{B}(k)$, although the IK spectrum recovers for strong turbulence.

Biskamp and Müller ${ }^{12}$ (hereafter, BM) and Müller and Grappin $^{13}$ (hereafter, MG) obtained $E(k) \propto k^{-5 / 3}$ in their recent direct numerical simulations (DNS) of decaying quasiisotropic incompressible MHD turbulence.

In this paper, we study the structures of isotropic incompressible MHD turbulence in the inertial subrange by making use of the Lagrangian renormalized approximation (LRA). ${ }^{14}$ We found that the total energy spectrum $E(k)$ is consistent with the IK spectrum, which confirmed the suggestion raised by Kaneda and Gotoh, ${ }^{15}$ and that the residual energy spectrum is given by the form $E^{R}(k)=A^{R} \epsilon B_{0}^{-1} k^{-2}$ with $A^{R}$ being a dimensionless constant, which is consistent with the result in Ref. 4. Furthermore, we obtained the numerical estimates of (i) the dimensionless constants $A$ and $A^{R}$, (ii) contribution of the triad interactions to the energy flux, and (iii) the eddy viscosity and the eddy magnetic diffusivity, without introducing any ad hoc parameters. In addition to the theoretical investigation, we performed DNS of a forced quasi-isotropic 
incompressible MHD turbulence, and compared the energy spectrum and the values of $A$ and $A^{R}$ obtained in the DNS with those derived in LRA.

LRA is one of the Lagrangian two-point closure approximations (LCAs). Here, LCAs refer to the abridged Lagrangian history direct interaction approximation (ALHDIA) ${ }^{16}$ and the strain-based abridged Lagrangian history direct interaction approximation (SBALHDIA) $)^{17}$ as well as LRA. The equations within LCAs are derived from the basic equations of fluids in systematic ways without any ad hoc parameters. Note that, within the EDQNM approximation, one has to introduce an ad hoc quantity, i.e., the eddy-damping rate. To the authors' knowledge, among various closure approximations, only three of the LCAs can provide the Kolmogorov spectrum $E^{u}(k)=C_{K} \epsilon^{2 / 3} k^{-5 / 3}$ for hydrodynamic turbulence with the value of $C_{K}$ being in good agreement with those in experiments and DNSs as well as the Kolmogorov scaling $k^{-2 / 3}$ for characteristic time in the inertial subrange. It is known that the closure based on quasi-Lagrangian variables ${ }^{18}$ also provides the Kolmogorov spectrum, however, to the authors' knowledge, the value of $C_{K}$ within the closure has not been estimated. Among LCAs, LRA is attractive in the sense that, for the hydrodynamic case, (i) the two-time correlation function and the response function satisfy the same equation of motion, which allows us to use formally the fluctuation-dissipation relation, and (ii) the structure of the equation of motion is simpler than the other LCAs.

There are pioneering studies on application of LRA to MHD turbulence by Kaneda and Gotoh ${ }^{15}$ and by Nakayama. ${ }^{19,20}$ Kaneda and Gotoh derived the IK spectrum for $E(k)$ in isotropic MHD turbulence. Nakayama studied MHD turbulence with strong uniform mean magnetic field $\mathbf{B}_{\mathrm{m}}$. Under a simplification that fluctuating velocity and magnetic fields are perpendicular to $\mathbf{B}_{\mathrm{m}}$, it was shown that $E\left(k_{\perp}\right) \propto k_{\perp}^{-3 / 2}$, where $k_{\perp}$ denotes the wave number perpendicular to $\mathbf{B}_{\mathrm{m}}$. Quantitative analyses such as the estimate of dimensionless constants were not made in these studies.

The DNS in the present study is of forced quasi-isotropic MHD turbulence in contrast to BM and MG, in which DNSs of decaying MHD turbulence were performed. Note that a substantial inertial subrange is necessary, i.e., the Reynolds number must be high enough, to test the present theoretical prediction of the energy spectrum. We forced the system in order to attain a Reynolds number as high as possible within the limitation of available computational resource. Both $\mathbf{u}$ and $\mathbf{B}$ were stirred at large scales by random force whose correlation time scale is of the order of the large-eddy turnover time.

\section{LRA EQUATIONS FOR MHD TURBULENCE} by

The basic equations of incompressible MHD are given

$$
\begin{aligned}
& \partial_{t} u_{i}+u_{j} \partial_{j} u_{i}=B_{j} \partial_{j} B_{i}-\partial_{i} P+\nu \partial_{j} \partial_{j} u_{i}, \\
& \partial_{i} u_{i}=0, \\
& \partial_{t} B_{i}+u_{j} \partial_{j} B_{i}=B_{j} \partial_{j} u_{i}+\lambda \partial_{j} \partial_{j} B_{i},
\end{aligned}
$$

$$
\partial_{i} B_{i}=0
$$

where $\mathbf{u}(\mathbf{x}, t)$ is the velocity field, $\mathbf{B}(\mathbf{x}, t)$ is an appropriately normalized magnetic field, $P(\mathbf{x}, t):=p(\mathbf{x}, t)+|\mathbf{B}(\mathbf{x}, t)|^{2} / 2$ is the total pressure, $p(\mathbf{x}, t)$ is the normal pressure, $\nu$ is the kinematic viscosity, and $\lambda$ is the magnetic diffusivity. The density of fluid is normalized to unity. Summation over $\{1,2,3\}$ for repeated indices is applied. In the following, we employ the notations

$$
X_{i}^{u}:=u_{i}, \quad X_{i}^{B}:=B_{i},
$$

for convenience.

Let us introduce the Lagrangian position function $\psi$ defined by

$$
\psi\left(\mathbf{x}^{\prime}, t ; \mathbf{x}, s\right):=\delta^{(3)}\left(\mathbf{x}^{\prime}-\mathbf{y}(\mathbf{x}, s \mid t)\right),
$$

where $\delta^{(3)}$ is the three-dimensional delta function and $\mathbf{y}(\mathbf{x}, s \mid t)$ is the position of a fluid element at time $t$ under the condition that the element was at $\mathbf{x}$ at time $s$. The function $\psi$ obeys

$$
\begin{aligned}
& \partial_{t} \psi\left(\mathbf{x}^{\prime}, t ; \mathbf{x}, s\right)=-u_{i}\left(\mathbf{x}^{\prime}, t\right) \partial_{i}^{\left(\mathbf{x}^{\prime}\right)} \psi\left(\mathbf{x}^{\prime}, t ; \mathbf{x}, s\right), \\
& \psi\left(\mathbf{x}^{\prime}, t ; \mathbf{x}, t\right)=\delta^{(3)}\left(\mathbf{x}^{\prime}-\mathbf{x}\right) .
\end{aligned}
$$

The Lagrangian variables are defined by

$$
X_{i}^{\alpha}(\mathbf{x}, s \mid t)=\int_{\mathcal{D}} d^{3} \mathbf{x}^{\prime} X_{i}^{\alpha}\left(\mathbf{x}^{\prime}, t\right) \psi\left(\mathbf{x}^{\prime}, t ; \mathbf{x}, s\right) \quad(t \geq s),
$$

where $\mathcal{D}$ is the domain of fluid and the upper Greek indices indicate $\{u, B\}$.

The two-point Lagrangian correlation function $Q$ and the Lagrangian response function $G$ are defined by

$Q_{i j}^{\alpha \beta}\left(\mathbf{x}, t ; \mathbf{x}^{\prime}, t^{\prime}\right):=\left\{\begin{array}{ll}\left\langle\left[\mathcal{P} X^{\alpha}\right]_{i}\left(\mathbf{x}, t^{\prime} \mid t\right) X_{j}^{\beta}\left(\mathbf{x}^{\prime}, t^{\prime}\right)\right\rangle & \left(t \geq t^{\prime}\right) \\ \left\langle X_{i}^{\alpha}(\mathbf{x}, t)\left[\mathcal{P} X^{\beta}\right]_{j}\left(\mathbf{x}^{\prime}, t \mid t^{\prime}\right)\right\rangle & \left(t<t^{\prime}\right)\end{array}\right.$,

$$
\begin{array}{r}
\left\langle\left[\mathcal{P} \delta X^{\alpha}\right]_{i}\left(\mathbf{x}, t^{\prime} \mid t\right)\right\rangle=G_{i j}^{\alpha \beta}\left(\mathbf{x}, t ; \mathbf{x}^{\prime}, t^{\prime}\right)\left[\mathcal{P} \delta X^{\beta}\right]_{j}\left(\mathbf{x}^{\prime}, t^{\prime} \mid t^{\prime}\right) \\
\left(t \geq t^{\prime}\right),
\end{array}
$$

where $\delta X_{j}^{\beta}\left(\mathbf{x}^{\prime}, t^{\prime} \mid t^{\prime}\right)$ is an infinitesimal disturbance added to $X_{j}^{\beta}\left(\mathbf{x}^{\prime}, t^{\prime} \mid t^{\prime}\right)$ at time $t^{\prime}, \delta X_{i}^{\alpha}\left(\mathbf{x}, t^{\prime} \mid t\right)$ is the response of $X_{i}^{\alpha}\left(\mathbf{x}, t^{\prime} \mid t\right)$ to the disturbance at time $t$, and $\mathcal{P} \mathbf{A}(\mathbf{x})$ is the projection of a vector field $\mathbf{A}(\mathbf{x})$ to its solenoidal part. $\langle Y\rangle$ denotes the average taken over an appropriate statistical ensemble for a quantity $Y$. Summation over $\{u, B\}$ for repeated upper Greek indices is applied.

We consider the case that the turbulence is statistically homogeneous, i.e., the statistically averaged quantities are invariant under the parallel translation $\left(\mathbf{x}^{(1)}, \ldots, \mathbf{x}^{(m)}\right)$ $\rightarrow\left(\mathbf{x}^{(1)}+\mathbf{r}, \ldots, \mathbf{x}^{(m)}+\mathbf{r}\right)$ of the reference points. When the turbulence is statistically homogeneous, $Q$ and $G$ depend on positions $\mathbf{x}$ and $\mathbf{x}^{\prime}$ only through $\mathbf{x}-\mathbf{x}^{\prime}$ and it is convenient to introduce the Fourier transforms of $Q$ and $G$ as 


$$
\begin{aligned}
\hat{Q}_{i j}^{\alpha \beta}\left(\mathbf{k}, t, t^{\prime}\right):= & (2 \pi)^{-3} \\
& \times \int d^{3}\left(\mathbf{x}-\mathbf{x}^{\prime}\right) e^{-i \mathbf{k} \cdot\left(\mathbf{x}-\mathbf{x}^{\prime}\right)} Q_{i j}^{\alpha \beta}\left(\mathbf{x}, t, \mathbf{x}^{\prime}, t^{\prime}\right), \\
\hat{G}_{i j}^{\alpha \beta}\left(\mathbf{k}, t, t^{\prime}\right):= & \int d^{3}\left(\mathbf{x}-\mathbf{x}^{\prime}\right) e^{-i \mathbf{k} \cdot\left(\mathbf{x}-\mathbf{x}^{\prime}\right)} G_{i j}^{\alpha \beta}\left(\mathbf{x}, t, \mathbf{x}^{\prime}, t^{\prime}\right),
\end{aligned}
$$

respectively. In the following, the hat ${ }^{\wedge}$ indicating the Fourier components is omitted unless it is ambiguous.

Following similar procedures to those given in Ref. 14, one obtains closed equations for $Q$ and $G$ as follows:

$$
\begin{aligned}
\partial_{t} Q_{i j}^{\alpha \beta}(\mathbf{k}, t, t)= & \int_{\mathbf{p}, \mathbf{q}}^{\triangle}\left[H_{i j}^{\alpha \beta}(\mathbf{k}, \mathbf{p}, \mathbf{q}, t)+H_{j i}^{\beta \alpha}(-\mathbf{k},-\mathbf{p},-\mathbf{q}, t)\right] \\
& -\nu^{\alpha \gamma} k^{2} Q_{i j}^{\gamma \beta}(\mathbf{k}, t, t)-\nu^{\beta \gamma} k^{2} Q_{i j}^{\alpha \gamma}(\mathbf{k}, t, t), \\
\partial_{t} Q_{i j}^{\alpha \beta}(\mathbf{k}, t, s)= & \int_{\mathbf{p}, \mathbf{q}}^{\triangle} I_{i j}^{\alpha \beta}(\mathbf{k}, \mathbf{p}, \mathbf{q}, t, s)-\nu^{\alpha \gamma} k^{2} Q_{i j}^{\gamma \beta}(\mathbf{k}, t, s) \\
\partial_{t} G_{i j}^{\alpha \beta}(\mathbf{k}, t, s)= & \int_{\mathbf{p}, \mathbf{q}}^{\triangle} J_{i j}^{\alpha \beta}(\mathbf{k}, \mathbf{p}, \mathbf{q}, t, s)-\nu^{\alpha \gamma} k^{2} G_{i j}^{\gamma \beta}(\mathbf{k}, t, s) \\
G_{i j}^{\alpha \beta}(\mathbf{k}, t, t)=P_{i j}(\mathbf{k}) \delta_{\alpha \beta}, \quad(2.14) & (t \geq s), \quad(2.16)
\end{aligned}
$$

where

$$
\begin{aligned}
H_{i j}^{\alpha \beta}(\mathbf{k}, \mathbf{p}, \mathbf{q}, t)= & 4 M_{i a b}^{\alpha \gamma \zeta}(\mathbf{k}) M_{c d e}^{\eta \kappa \xi}(\mathbf{p}) \\
& \times \int_{t_{0}}^{t} d s G_{a c}^{\gamma \eta}(\mathbf{p}, t, s) Q_{b d}^{\zeta \kappa}(\mathbf{q}, t, s) Q_{j e}^{\beta \xi}(-\mathbf{k}, t, s) \\
& +2 M_{i a b}^{\alpha \gamma \zeta}(\mathbf{k}) M_{e c d}^{\xi \eta \kappa}(-\mathbf{k}) \\
& \times \int_{t_{0}}^{t} d s Q_{a c}^{\gamma \eta}(\mathbf{p}, t, s) Q_{b d}^{\zeta \kappa}(\mathbf{q}, t, s) G_{j e}^{\beta \xi}(-\mathbf{k}, t, s)
\end{aligned}
$$

$$
\begin{aligned}
I_{i j}^{\alpha \beta}(\mathbf{k}, \mathbf{p}, \mathbf{q}, t, s) & \\
= & 4 P_{i l}(\mathbf{k}) N_{l a b}^{\alpha \gamma \zeta}(\mathbf{k}) M_{c d e}^{\eta \kappa \xi}(\mathbf{p}) \\
& \times \int_{t_{0}}^{t} d s^{\prime} G_{a c}^{\gamma \eta}\left(\mathbf{p}, t, s^{\prime}\right) Q_{b d}^{\zeta \kappa}\left(\mathbf{q}, t, s^{\prime}\right) Q_{e j}^{\xi \beta}\left(\mathbf{k}, s^{\prime}, s\right) \\
& +2 P_{i l}(\mathbf{k}) N_{l a b}^{\alpha \gamma \zeta}(\mathbf{k}) M_{e c d}^{\xi \eta \kappa}(-\mathbf{k}) \\
& \times \int_{t_{0}}^{s} d s^{\prime} Q_{a c}^{\gamma \eta}\left(\mathbf{p}, t, s^{\prime}\right) Q_{b d}^{\zeta \kappa}\left(\mathbf{q}, t, s^{\prime}\right) G_{j e}^{\beta \xi}\left(-\mathbf{k}, s, s^{\prime}\right) \\
& +2 P_{i l}(\mathbf{k}) N_{l a b}^{\alpha \gamma \zeta}(\mathbf{p}) A_{c}^{u}(-\mathbf{p}) \\
& \times \int_{s}^{t} d s^{\prime} Q_{b c}^{\zeta u}\left(-\mathbf{q}, t, s^{\prime}\right) Q_{a j}^{\gamma \beta}(\mathbf{k}, t, s)
\end{aligned}
$$

$$
\begin{aligned}
J_{i j}^{\alpha \beta}(\mathbf{k}, \mathbf{p}, \mathbf{q}, t, s)= & 4 P_{i l}(\mathbf{k}) N_{l a b}^{\alpha \gamma \zeta}(\mathbf{k}) M_{c d e}^{\eta \kappa \xi}(\mathbf{p}) \\
& \times \int_{s}^{t} d s^{\prime} G_{a c}^{\gamma \eta}\left(\mathbf{p}, t, s^{\prime}\right) Q_{b d}^{\zeta \kappa}\left(\mathbf{q}, t, s^{\prime}\right) G_{e j}^{\xi \beta}\left(\mathbf{k}, s^{\prime}, s\right) \\
& +2 P_{i l}(\mathbf{k}) N_{l a b}^{\alpha \gamma \zeta}(\mathbf{p}) A_{c}^{u}(-\mathbf{p}) \\
& \times \int_{s}^{t} d s^{\prime} Q_{b c}^{\zeta u}\left(-\mathbf{q}, t, s^{\prime}\right) G_{a j}^{\gamma \beta}(\mathbf{k}, t, s)
\end{aligned}
$$

with

$$
\begin{aligned}
& M_{i m n}^{u u u}(\mathbf{k})=-\frac{i}{2}\left[k_{m} P_{i n}(\mathbf{k})+k_{n} P_{i m}(\mathbf{k})\right], \\
& M_{i m n}^{u B B}(\mathbf{k})=\frac{i}{2}\left[k_{m} P_{i n}(\mathbf{k})+k_{n} P_{i m}(\mathbf{k})\right], \\
& M_{i m n}^{B u B}(\mathbf{k})=\frac{i}{2}\left(\delta_{i m} k_{n}-\delta_{i n} k_{m}\right), \\
& M_{i m n}^{B B u}(\mathbf{k})=\frac{i}{2}\left(-\delta_{i m} k_{n}+\delta_{i n} k_{m}\right), \\
& M_{i m n}^{u u B}(\mathbf{k})=M_{i m n}^{u B u}(\mathbf{k})=M_{i m n}^{B u u}(\mathbf{k})=M_{i m n}^{B B B}(\mathbf{k})=0, \\
& N_{i m n}^{u u u}(\mathbf{k})= \\
& N_{i m n}^{u u B}(\mathbf{k})=N_{i m n}^{u B u}(\mathbf{k})=N_{i m n}^{B u u}(\mathbf{k})=N_{i m n}^{B B B}(\mathbf{k})=0, \\
& k^{2}(\mathbf{k})=-i k_{i}, \\
& N_{i m n}^{B B u}(\mathbf{k})=\frac{i}{2} \delta_{i n} k_{m}, \\
& N_{i m n}^{B u B}(\mathbf{k})=\frac{i}{2} \delta_{i m} k_{n}, \\
& \nu^{u}(\mathbf{k})=\frac{i}{2}\left[k_{m} P_{i n}(\mathbf{k})+k_{n} P_{i m}(\mathbf{k})\right],
\end{aligned}
$$

and

$$
\begin{aligned}
P_{i j}(\mathbf{k}) & :=\delta_{i j}-\frac{k_{i} k_{j}}{k^{2}}, \quad k:=|\mathbf{k}|, \\
\int_{\mathbf{p}, \mathbf{q}}^{\triangle} & :=\int d^{3} \mathbf{p} \int d^{3} \mathbf{q} \delta^{(3)}(\mathbf{k}-\mathbf{p}-\mathbf{q}) .
\end{aligned}
$$

Here, $t_{0}$ is the initial time of the integration.

\section{EQUATIONS FOR ISOTROPIC TURBULENCE}

The MHD equations (2.1)-(2.4) are invariant under spatial translations, rotations, and the parity transformations $\mathbf{x}$ $\rightarrow-\mathbf{x}, \mathbf{u} \rightarrow-\mathbf{u}, \mathbf{B} \rightarrow \pm \mathbf{B}$. When statistically averaged quantities are invariant under all of these transformations, the 
MHD turbulence is called homogeneous and isotropic. In the case of homogeneous and isotropic MHD turbulence, $Q$ and $G$ may be written in the following forms:

$$
\begin{aligned}
& Q_{i j}^{u u}(\mathbf{k}, t, s)=\frac{1}{2} Q^{u}(k, t, s) P_{i j}(\mathbf{k}), \\
& Q_{i j}^{B B}(\mathbf{k}, t, s)=\frac{1}{2} Q^{B}(k, t, s) P_{i j}(\mathbf{k}), \\
& Q_{i j}^{u B}(\mathbf{k}, t, s)=Q_{i j}^{B u}(\mathbf{k}, t, s)=0, \\
& G_{i j}^{u u}(\mathbf{k}, t, s)=G^{u}(k, t, s) P_{i j}(\mathbf{k}), \\
& G_{i j}^{B B}(\mathbf{k}, t, s)=G^{B}(k, t, s) P_{i j}(\mathbf{k}), \\
& G_{i j}^{u B}(\mathbf{k}, t, s)=G_{i j}^{B u}(\mathbf{k}, t, s)=0 .
\end{aligned}
$$

Then, the LRA equations (2.14)-(2.17) reduce to

$\left[\partial_{t}+2 \nu^{\alpha} k^{2}\right] Q^{\alpha}(k, t, t)=4 \pi \iint_{\triangle} d p d q \frac{p q}{k} H^{\alpha}(k, p, q ; t)$,

$\left[\partial_{t}+\nu^{\alpha} k^{2}\right] Q^{\alpha}(k, t, s)=2 \pi \iint_{\triangle} d p d q \frac{p q}{k} I^{\alpha}(k, p, q ; t, s)$,

$\left[\partial_{t}+\nu^{\alpha} k^{2}\right] G^{\alpha}(k, t, s)=2 \pi \iint_{\triangle} d p d q \frac{p q}{k} J^{\alpha}(k, p, q ; t, s)$,

$G^{\alpha}(k, t, t)=1$,

where

$$
\begin{aligned}
H^{u}(k, p, q ; t)= & -\frac{1}{2} k p\left(x y+z^{3}\right) \\
& \times \int_{t_{0}}^{t} d s G^{u}(p, t, s) Q^{u}(q, t, s) Q^{u}(k, t, s) \\
& -\frac{1}{2} k p\left(1-y^{2}\right) z \\
& \times \int_{t_{0}}^{t} d s G^{B}(p, t, s) Q^{B}(q, t, s) Q^{u}(k, t, s) \\
& +\frac{1}{8} k^{2}\left(1+x^{2}+y^{2}+z^{2}-4 y^{2} z^{2}\right) \\
& \times \int_{t_{0}}^{t} d s Q^{u}(p, t, s) Q^{u}(q, t, s) G^{u}(k, t, s) \\
& +\frac{1}{8} k^{2}\left(1+x^{2}+y^{2}+z^{2}-4 y^{2} z^{2}\right) \\
& \times \int_{t_{0}}^{t} d s Q^{B}(p, t, s) Q^{B}(q, t, s) G^{u}(k, t, s),
\end{aligned}
$$

$$
\begin{aligned}
H^{B}(k, p, q ; t)= & -\frac{1}{2} k p\left(1-x^{2}\right) z \\
& \times \int_{t_{0}}^{t} d s G^{u}(p, t, s) Q^{B}(q, t, s) Q^{B}(k, t, s) \\
& -\frac{1}{2} k p(x y+z) \\
& \times \int_{t_{0}}^{t} d s G^{B}(p, t, s) Q^{u}(q, t, s) Q^{B}(k, t, s) \\
& +\frac{1}{4} k^{2}\left(3-x^{2}-y^{2}-z^{2}\right) \\
& \times \int_{t_{0}}^{t} d s Q^{u}(p, t, s) Q^{B}(q, t, s) G^{B}(k, t, s) \\
I^{u}(k, p, q ; t, s)= & -\frac{1}{2} k p\left(1-y^{2}\right) z \\
& \times \int_{t_{0}}^{t} d s^{\prime} G^{B}\left(p, t, s^{\prime}\right) Q^{B}\left(q, t, s^{\prime}\right) Q^{u}\left(k, s^{\prime}, s\right) \\
& +\frac{1}{8} k^{2}\left(1+x^{2}+y^{2}+z^{2}-4 y^{2} z^{2}\right) \\
& \times \int_{t_{0}}^{s} Q^{B}\left(p, t, s^{\prime}\right) Q^{B}\left(q, t, s^{\prime}\right) G^{u}\left(k, s, s^{\prime}\right) \\
& -\frac{1}{2} k^{2}\left(1-y^{2}\right)\left(1-z^{2}\right) \\
& \times \int^{\prime} Q^{u}\left(q, t, s^{\prime}\right) Q^{u}(k, t, s) \\
& \\
&
\end{aligned}
$$

$$
\begin{aligned}
I^{B}(k, p, q ; t, s)= & -\frac{1}{4} k p\left(x y+z-x^{2} z+z^{3}\right) \\
& \times \int_{t_{0}}^{t} d s^{\prime} G^{u}\left(p, t, s^{\prime}\right) Q^{B}\left(q, t, s^{\prime}\right) Q^{B}\left(k, s^{\prime}, s\right) \\
& -\frac{1}{4} k p y(x+y z) \\
& \times \int_{t_{0}}^{t} d s^{\prime} G^{B}\left(p, t, s^{\prime}\right) Q^{u}\left(q, t, s^{\prime}\right) Q^{B}\left(k, s^{\prime}, s\right) \\
& +\frac{1}{8} k^{2}\left(3-x^{2}-3 y^{2}+z^{2}\right) \\
& \times \int_{t_{0}}^{s} d s^{\prime} Q^{u}\left(p, t, s^{\prime}\right) Q^{B}\left(q, t, s^{\prime}\right) G^{B}\left(k, s, s^{\prime}\right) \\
& -\frac{1}{8} p^{2}\left(1-x^{2}+y^{2}-z^{2}\right) \\
& \times \int_{s}^{t} d s^{\prime} Q^{u}\left(q, t, s^{\prime}\right) Q^{B}(k, t, s),
\end{aligned}
$$




$$
\begin{aligned}
J^{u}(k, p, q ; t, s)= & -\frac{1}{2} k p\left(1-y^{2}\right) z \\
& \times \int_{s}^{t} d s^{\prime} G^{B}\left(p, t, s^{\prime}\right) Q^{B}\left(q, t, s^{\prime}\right) G^{u}\left(k, s^{\prime}, s\right) \\
& -\frac{1}{2} k^{2}\left(1-y^{2}\right)\left(1-z^{2}\right) \\
& \times \int_{s}^{t} d s^{\prime} Q^{u}\left(q, t, s^{\prime}\right) G^{u}(k, t, s), \\
J^{B}(k, p, q ; t, s)= & -\frac{1}{4} k p\left(x y+z-x^{2} z+z^{3}\right) \\
& \times \int_{s}^{t} d s^{\prime} Q^{B}\left(q, t, s^{\prime}\right) G^{u}\left(p, t, s^{\prime}\right) G^{B}\left(k, s^{\prime}, s\right) \\
& -\frac{1}{4} k p y(x+y z) \\
& \times \int_{s}^{t} d s^{\prime} G^{B}\left(p, t, s^{\prime}\right) Q^{u}\left(q, t, s^{\prime}\right) G^{B}\left(k, s^{\prime}, s\right) \\
& -\frac{1}{8} p^{2}\left(1-x^{2}+y^{2}-z^{2}\right) \\
& \times \int_{s}^{t} d s^{\prime} Q^{u}\left(q, t, s^{\prime}\right) G^{B}(k, t, s),
\end{aligned}
$$

with

$$
x:=-\frac{\mathbf{p} \cdot \mathbf{q}}{p q}, \quad y:=\frac{\mathbf{q} \cdot \mathbf{k}}{q k}, \quad z:=\frac{\mathbf{k} \cdot \mathbf{p}}{k p}
$$

and

$$
\iint_{\triangle} d p d q:=\int_{0}^{\infty} d p \int_{|p-k|}^{p+k} d q
$$

In the derivation, we used the identities

$$
x y z=\frac{1}{2}\left(1-x^{2}-y^{2}-z^{2}\right), \quad p^{2}\left(1-x^{2}\right)=k^{2}\left(1-y^{2}\right) .
$$

The tensorial algebras were performed by using MATHEMATICA. [Expressions equivalent to (3.7)-(3.16) were obtained in Ref. 15 for the first time, but some geometric coefficients are given incorrectly.]

The kinetic energy spectrum $E^{u}(k, t)$ and the magnetic energy spectrum $E^{B}(k, t)$ are given, respectively, in terms of $Q$ by

$$
\begin{aligned}
& E^{u}(k, t)=2 \pi k^{2} Q^{u}(k, t, t), \\
& E^{B}(k, t)=2 \pi k^{2} Q^{B}(k, t, t) .
\end{aligned}
$$

The total energy flux $\Pi(k, t)$ flowing into wave numbers larger than $k$ may be written as

$$
\Pi(k, t)=\frac{1}{2} \int_{k}^{\infty} d k^{\prime} \int_{0}^{\infty} d p^{\prime} \int_{\left|p^{\prime}-k^{\prime}\right|}^{p^{\prime}+k^{\prime}} d q^{\prime} T\left(k^{\prime}, p^{\prime}, q^{\prime} ; t\right),
$$

where

$$
T(k, p, q ; t)=16 \pi^{2} k p q\left[H^{u}(k, p, q ; t)+H^{B}(k, p, q ; t)\right]
$$

[cf. (3.7) in Ref. 21].

Let us introduce

$$
H_{i j}^{\alpha \beta>}\left(\mathbf{k}, k_{c}, t\right):=\int_{\mathbf{p}, \mathbf{q}}^{\Delta>k_{c}} H_{i j}^{\alpha \beta}(\mathbf{k}, \mathbf{p}, \mathbf{q} ; t),
$$

where $\int_{\mathbf{p}, \mathbf{q}}^{\Delta>k_{c}}$ denotes the integral over $\mathbf{p}$ and $\mathbf{q}$ satisfying $\mathbf{p}$ $+\mathbf{q}=\mathbf{k}$, and $p$ and/or $q>k_{c}$. With the assumptions that $Q_{i j}^{\alpha \beta}(\mathbf{k}, t, s)$ and $G_{i j}^{\alpha \beta}(\mathbf{k}, t, s)$ are isotropic for $k>k_{c}$, and that the amplitude of $Q^{\alpha}(k, t, s)$ and the scales of characteristic decay time for $Q^{\alpha}(k, t, s)$ and $G^{\alpha}(k, t, s)$ decrease sufficiently fast as $k$ increases, the asymptotic form of $H_{i j}^{\alpha \beta>}\left(\mathbf{k}, k_{c}, t\right)$ for $k \ll k_{c}$ is given by

$$
H_{i j}^{\alpha \beta>}\left(\mathbf{k}, k_{c}, t\right)=-\nu^{\alpha \gamma}\left(k_{c}, t\right) k^{2} Q_{i j}^{\gamma \beta}(\mathbf{k}, t),
$$

where

$$
\begin{aligned}
\nu^{u u}\left(k_{c}, t\right)= & \frac{2 \pi}{15} \int_{k_{c}}^{\infty} d p \int_{t_{0}}^{t} d s\left\{7 p^{2}\left[G^{u}(p, t, s)\right]^{2} Q^{u}(p, s, s)\right. \\
& +7 p^{2}\left[G^{B}(p, t, s)\right]^{2} Q^{B}(p, s, s) \\
& +p^{3}\left[G^{u}(p, t, s)\right]^{2}\left(\frac{\partial}{\partial q} Q^{u}(q, s, s)\right)_{q=p} \\
& \left.-p^{3}\left(G^{B}(p, t, s)\right)^{2}\left(\frac{\partial}{\partial q} Q^{B}(q, s, s)\right)_{q=p}\right\}
\end{aligned}
$$

$\nu^{B B}\left(k_{c}, t\right)=\frac{4 \pi}{3} \int_{k_{c}}^{\infty} d p \int_{t_{0}}^{t} d s p^{2} G^{u}(p, t, s) G^{B}(p, t, s) Q^{u}(p, s, s)$,

$\nu^{u B}\left(k_{c}, t\right)=\nu^{B u}\left(k_{c}, t\right)=0$.

Note that $Q$ and $G$ are not necessarily isotropic for $k<k_{c}$. The result (3.25) with (3.26)-(3.28) implies that the effect of wave numbers higher than $k_{c}$ on the time evolution of $Q_{i j}^{\alpha \beta}(\mathbf{k}, t, t)$ given by (2.14) can be renormalized in the form of the eddy viscosity $\nu^{u u}\left(k_{c}, t\right)$ and eddy magnetic diffusivity $\nu^{B B}\left(k_{c}, t\right)$, provided that $k / k_{c} \ll 1$.

When $Q$ and $G$ are isotropic in the entire wave-number range under consideration, one may formally introduce $k$-dependent eddy viscosity $\nu^{u}\left(k, k_{c}, t\right)$ and eddy magnetic diffusivity $\nu^{B}\left(k, k_{c}, t\right)$ for $k / k_{c}<1$ as

$$
\nu^{u}\left(k, k_{c}, t\right)=-\frac{H_{i i}^{u u>}\left(\mathbf{k}, k_{c}, t\right)}{k^{2} Q^{u}(k, t)},
$$




$$
\nu^{B}\left(k, k_{c}, t\right)=-\frac{H_{i i}^{B B>}\left(\mathbf{k}, k_{c}, t\right)}{k^{2} Q^{B}(k, t)},
$$

following Kraichnan's definition of the eddy viscosity. ${ }^{22} \mathrm{We}$ see that $\nu^{u}\left(k, k_{c}, t\right) \sim \nu^{u u}\left(k_{c}, t\right)$ and $\nu^{B}\left(k, k_{c}, t\right) \sim \nu^{B B}\left(k_{c}, t\right)$ for $k / k_{c} \ll 1$.

\section{INERTIAL SUBRANGE STRUCTURES}

We assume that statistically averaged quantities are homogeneous, isotropic, and stationary in the inertial subrange. In the following analysis, we put $t_{0}=-\infty$ in order to work with the statistically stationary state and we put $\nu=\lambda=0$ since viscosity and magnetic diffusivity in the closure equations may be neglected formally in the inertial subrange.

Let $k_{0}$ be the lower-boundary wave number of the inertial subrange. When

$$
Q^{u}(k, t, t) \sim k^{a^{u}}, \quad Q^{B}(k, t, t) \sim k^{a^{B}}
$$

are satisfied in the inertial subrange, it is easily seen that the integrals on the right-hand sides of (3.8) and (3.9) diverge like $k_{0}^{3+a^{B}}$ as $k_{0}$ tends to 0 provided that

(D1) $a^{B}<-3$, and that

(D2) the characteristic decay time of $Q^{B}\left(k_{1}, t, s\right)$ is much larger than those of $Q^{\alpha}\left(k_{2}, t, s\right)$ and $G^{\alpha}\left(k_{2}, t, s\right)(\alpha$ $=u, B)$ for $k_{1} \ll k_{2}$.

Note that the conditions (D1) and (D2) are satisfied when the Kolmogorov scaling is applied to $Q^{u}, G^{u}, Q^{B}$ and $G^{B}$ since $a^{u}=a^{B}=-11 / 3$ and the characteristic decay times of $Q^{u}(k)$ and $G^{u}(k)$ are proportional to $k^{-2 / 3}$ in the Kolmogorov scaling. The conditions are also satisfied for the IK phenomenology in which $a^{u}=a^{B}=-7 / 2$ and the characteristic decay times of $Q^{u}(k), G^{u}(k), Q^{B}(k)$ and $G^{B}(k)$ are proportional to $k^{-1}$.

In the direct interaction approximation (DIA) for MHD turbulence, both $Q^{u}$ and $Q^{B}$ contribute to the divergence in the integrals, ${ }^{3}$ while, in LRA, only $Q^{B}$ contributes to the divergence. Note that DIA is an Eulerian closure approximation. The divergence of the integrals due to $Q^{u}$, which appears in DIA, is removed in LRA by closing the equations with the Lagrangian variables, while the divergence due to $Q^{B}$ is not removed by the same procedure. The former divergence can be attributed to the inclusion of the sweeping effect of the eddies in the energy containing range on the eddies in the inertial subrange in the estimation of the energy transfer among the latter eddies. This is inappropriate since the sweeping effect can be removed by the Galilean transformation and the energy transfer should be evaluated on the frame moving with each eddy. In the purely hydrodynamic case, the closure equations result in an incorrect scaling $E^{u}(k) \propto k^{-3 / 2}$ when there is the divergence due to $Q^{u}$, whereas they result in the Kolmogorov scaling $E^{u}(k) \propto k^{-5 / 3}$ when the divergence is removed (see, e.g., Ref. 23). On the other hand, since there is no transformation of coordinate that removes a uniform magnetic field, the large scales of magnetic field in the energy-containing range may contribute reasonably to the energy transfer between smaller scales in the inertial subrange.
The divergence of the integrals in (3.8) and (3.9) implies that the main contribution of the integrals comes from small $p$ and $q\left(\leq k_{0}\right)$. Therefore, the integrand of (3.8) and (3.9) may be approximated by the leading orders in their Taylor expansions around $p=0$ with respect to $p / k$ and those around $q=0$ with respect to $q / k$. Resulting equations are

$$
\begin{aligned}
\partial_{t} Q^{u}(k, t, s)= & -B_{0}^{2} k^{2}\left\{\int_{s}^{t} d s^{\prime} G^{B}\left(k, t, s^{\prime}\right) Q^{u}\left(k, s^{\prime}, s\right)\right. \\
& +\int_{-\infty}^{s} d s^{\prime}\left[G^{B}\left(k, t, s^{\prime}\right) Q^{u}\left(k, s, s^{\prime}\right)\right. \\
& \left.\left.-G^{u}\left(k, s, s^{\prime}\right) Q^{B}\left(k, t, s^{\prime}\right)\right]\right\}, \\
\partial_{t} Q^{B}(k, t, s)= & -B_{0}^{2} k^{2}\left\{\int_{s}^{t} d s^{\prime} G^{u}\left(k, t, s^{\prime}\right) Q^{B}\left(k, s^{\prime}, s\right)\right. \\
& +\int_{-\infty}^{s} d s^{\prime}\left[G^{u}\left(k, t, s^{\prime}\right) Q^{B}\left(k, s, s^{\prime}\right)\right. \\
& \left.\left.-G^{B}\left(k, s, s^{\prime}\right) Q^{u}\left(k, t, s^{\prime}\right)\right]\right\}, \\
\partial_{t} G^{u}(k, t, s)= & -B_{0}^{2} k^{2} \int_{s}^{t} d s^{\prime} G^{B}\left(k, t, s^{\prime}\right) G^{u}\left(k, s^{\prime}, s\right), \\
\partial_{t} G^{B}(k, t, s)= & -B_{0}^{2} k^{2} \int_{s}^{t} d s^{\prime} G^{u}\left(k, t, s^{\prime}\right) G^{B}\left(k, s^{\prime}, s\right),
\end{aligned}
$$

where

$$
B_{0}:=\sqrt{\left\langle B_{i}^{2}\right\rangle}=\sqrt{\frac{2}{3} \int_{0}^{\infty} d k E^{B}(k)},
$$

with $i$ being any of the coordinate indices $\{1,2,3\}$.

Equations (4.4) and (4.5) with (3.10) have a solution of a similarity form

$$
G^{u}(k, t, s)=G^{B}(k, t, s)=g\left(B_{0} k(t-s)\right),
$$

with

$$
g(\tau)=\frac{J_{1}(2 \tau)}{\tau},
$$

where $J_{1}(\tau)$ is the Bessel function of the first kind of the order 1 . The rescaled response function $g(\tau)$ is plotted in Fig. 1. In the following, we will omit the superscripts $u$ and $B$ on $G$.

It is convenient to introduce $Q^{E}$ and $Q^{R}$ defined by

$$
\begin{aligned}
& Q^{E}(k, t, s):=\frac{1}{2}\left[Q^{u}(k, t, s)+Q^{B}(k, t, s)\right], \\
& Q^{R}(k, t, s):=Q^{u}(k, t, s)-Q^{B}(k, t, s) .
\end{aligned}
$$

They are related to the total energy spectrum $E(k, t)$ and the residual energy spectrum $E^{R}(k, t)$ by

$$
E(k, t)=4 \pi k^{2} Q^{E}(k, t, t),
$$




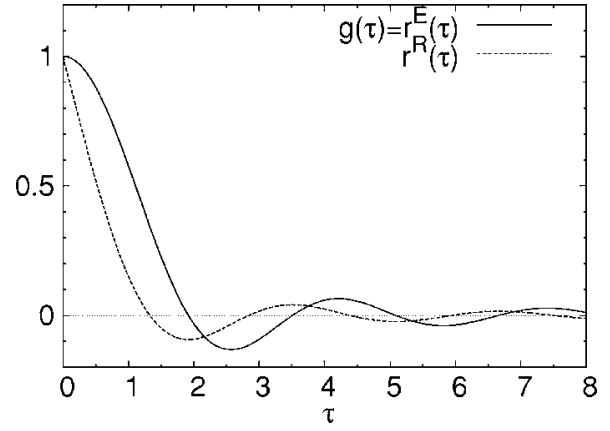

FIG. 1. Rescaled response function $g$, and rescaled two-time correlation functions $r^{E}$ and $r^{R}$.

$$
E^{R}(k, t)=2 \pi k^{2} Q^{R}(k, t, t) .
$$

From (4.2), (4.3), (4.9), and (4.10), one obtains

$$
\begin{aligned}
\partial_{t} Q^{E}(k, t, s)= & -B_{0}^{2} k^{2}\left\{\int_{s}^{t} d s^{\prime} G\left(k, t, s^{\prime}\right) Q^{E}\left(k, s^{\prime}, s\right)\right. \\
& +\int_{-\infty}^{s} d s^{\prime}\left[G\left(k, t, s^{\prime}\right) Q^{E}\left(k, s, s^{\prime}\right)\right. \\
& \left.\left.-G\left(k, s, s^{\prime}\right) Q^{E}\left(k, t, s^{\prime}\right)\right]\right\}, \\
\partial_{t} Q^{R}(k, t, s)= & -B_{0}^{2} k^{2}\left\{\int_{s}^{t} d s^{\prime} G\left(k, t, s^{\prime}\right) Q^{R}\left(k, s^{\prime}, s\right)\right. \\
& +\int_{-\infty}^{s} d s^{\prime}\left[G\left(k, t, s^{\prime}\right) Q^{R}\left(k, s, s^{\prime}\right)\right. \\
& \left.\left.+G\left(k, s, s^{\prime}\right) Q^{R}\left(k, t, s^{\prime}\right)\right]\right\} .
\end{aligned}
$$

Equations (4.13) and (4.14) have a solution of similarity forms

$$
\begin{aligned}
& Q^{E}(k, t, s)=Q^{E}(k) r^{E}\left(B_{0} k(t-s)\right), \\
& Q^{R}(k, t, s)=Q^{R}(k) r^{R}\left(B_{0} k(t-s)\right),
\end{aligned}
$$

where

$$
r^{E}(\tau)=\frac{J_{1}(2 \tau)}{\tau}[=g(\tau)]
$$

and $r^{R}(\tau)$ is the solution of the equation

$$
\begin{aligned}
\partial_{t} r^{R}(\tau)= & -\int_{0}^{\tau} d \sigma g(\tau-\sigma) r^{R}(\sigma) \\
& -\int_{0}^{\infty} d \sigma\left[g(\tau+\sigma) r^{R}(\sigma)+g(\sigma) r^{R}(\tau+\sigma)\right],
\end{aligned}
$$

with $r^{R}(0)=1$.

We assume that the total energy flux $\Pi(k)$ is constant in the inertial subrange and put it equal to the energy dissipation rate $\epsilon$ independent of $k$, i.e.,

$$
\Pi(k)=\epsilon .
$$

Let us further assume that $Q^{E}(k)$ and $Q^{R}(k)$ in (4.15) and (4.16), respectively, are given by the similarity forms

$$
\begin{aligned}
& Q^{E}(k)=\frac{A}{2 \pi} \epsilon^{b^{E}} B_{0}^{c^{E}} k^{a^{E}}, \\
& Q^{R}(k)=\frac{A^{R}}{2 \pi} \epsilon^{b^{R}} B_{0}^{c^{R}} k^{a^{R}},
\end{aligned}
$$

with

$$
a^{E}>a^{R}
$$

where $A$ and $A^{R}$ are dimensionless constants. The assumption (4.22) implies that $Q^{E}(k) \gg Q^{R}(k)$, i.e., $Q^{u}(k) \sim Q^{B}(k)$, at sufficiently high $k$. It will be checked later that the assumption is consistent with the result.

By using (3.11), (3.12), (3.22), (3.23), (4.7)-(4.10), and (4.15)-(4.17), the constant energy flux condition (4.19) reduces to

$$
\boldsymbol{\epsilon}=A^{2} K \boldsymbol{\epsilon}^{2 b^{E}} B_{0}^{-1+2 c^{E}} k^{7+2 a^{E}}+O\left(k^{7+a^{E}+a^{R}}\right) \quad(k \rightarrow \infty),
$$

where $K$ is a constant. Note that, by virtue of the assumption (4.22), $Q^{R}$ do not contribute to the leading order of the energy flux for sufficiently high $k$. By neglecting the terms of $O\left(k^{7+a^{E}+a^{R}}\right)$ in (4.23), one obtains

$$
a^{E}=-\frac{7}{2}, \quad b^{E}=\frac{1}{2}, \quad c^{E}=\frac{1}{2}, \quad A=K^{-1 / 2} .
$$

The constant $K$ is given by

$$
K=\int_{0}^{1} d v \ln \left(\frac{1}{v}\right) \int_{\max (v, 1-v)}^{v+1} d w \tilde{T}(1, v, w),
$$

where $\tilde{T}(k, p, q)$ is obtained by substituting (3.11), (3.12), (4.7), (4.8), (4.15), (4.17), and (4.20) and $Q^{u}(k, t, s)$ $=Q^{B}(k, t, s)=Q^{E}(k, t, s)$ into $T(k, p, q)$ of (3.23), and then putting $\epsilon, B_{0}$, and $A$ to unity.

To the leading order of $k$, we may write (4.19) with (3.22) in the following form:

$$
\epsilon=\int_{1}^{\infty} \frac{d \alpha}{\alpha} W(\alpha),
$$

with

$$
\begin{aligned}
W(\alpha)= & \alpha^{-1} \ln \alpha \int_{1}^{\min (\alpha, \alpha /(\alpha-1))} d \beta \beta^{-2} T\left(1, \frac{1}{\alpha}, \frac{1}{\beta}\right) \\
& +\alpha \int_{\max (1, \alpha-1)}^{\alpha} d \beta\left(\beta^{-3} \ln \beta\right) T\left(1, \frac{1}{\beta}, \frac{\alpha}{\beta}\right),
\end{aligned}
$$

where $\alpha$ represents the ratio of maximum to minimum wave numbers, i.e., 


$$
\alpha:=\frac{\max \left(k^{\prime}, p^{\prime}, q^{\prime}\right)}{\min \left(k^{\prime}, p^{\prime}, q^{\prime}\right)},
$$

of the triad $\left(\mathbf{k}^{\prime}, \mathbf{p}^{\prime}, \mathbf{q}^{\prime}\right)$ satisfying $k^{\prime}>k$ and $\mathbf{k}^{\prime}=\mathbf{p}^{\prime}+\mathbf{q}^{\prime}$. The integrand in (4.26), $W(\alpha) d \ln \alpha$, can be interpreted as the contribution of triad interactions to $\epsilon$ from the domain between $\ln \alpha$ and $\ln \alpha+d \ln \alpha$ [cf. (40) and (41) in Ref. 24].

The constant total energy flux condition (4.19) implies the stationarity of $Q^{u}(k, t, t)+Q^{B}(k, t, t)$. In order that each of $Q^{u}(k, t, t)$ and $Q^{B}(k, t, t)$ is stationary as well as their sum, it is required that

$$
H^{\alpha(0)}(k, t)+H^{\alpha(1)}(k, t)+H^{\alpha(2)}(k, t)=0,
$$

where $H^{\alpha(i)}(k, t)$ is the $i$ th order term in the power expansion of the right-hand side of (3.7) in $Q^{R}$. It can be shown that the integral over the wave number in $H^{\alpha(0)}(k, t)$ converges when the similarity forms of $Q$ and $G$ are substituted and that

$$
H^{u(0)}(k, t)=-H^{B(0)}(k, t)=A^{2} K_{1} k^{-3} \epsilon,
$$

where $K_{1}$ is a constant. On the other hand, the integrals over the wave number in $H^{\alpha(1)}(k, t)$ and $H^{\alpha(2)}(k, t)$ diverge as the lower-boundary wave number $k_{0}$ of the inertial subrange tends to 0 . A similar approximation to that in (4.2)-(4.5) leads to

$$
\begin{aligned}
H^{u(1)}(k, t)+H^{u(2)}(k, t) & =-H^{B(1)}(k, t)-H^{B(2)}(k, t) \\
& =-2 B_{0}^{2} k^{2} \int_{-\infty}^{t} d s G(k, t, s) Q^{R}(k, t, s) .
\end{aligned}
$$

From (4.7), (4.8), (4.16), (4.21), and (4.29)-(4.31), one obtains

$$
\begin{aligned}
& a^{R}=-4, \quad b^{R}=1, \quad c^{R}=-1, \\
& A^{R}=\pi A^{2} K_{1} I_{G R}^{-1},
\end{aligned}
$$

where

$$
I_{G R}:=\int_{0}^{\infty} d \tau g(\tau) r^{R}(\tau)
$$

The results (4.24) and (4.32) are consistent with the assumption (4.22).

From (4.20), (4.21), (4.24), and (4.32), one gets $Q^{R}(k) / Q^{E}(k) \propto \Delta(k)$, where

$$
\Delta(k):=\epsilon^{1 / 2} B_{0}^{-3 / 2} k^{-1 / 2} .
$$

The present analysis is valid in the wave-number range where $\Delta(k) \ll 1$, i.e., $k \gg k_{B}$ with

$$
k_{B}:=\epsilon B_{0}^{-3} \text {. }
$$

In this wave-number range, $Q^{u}(k) \sim Q^{B}(k) \sim Q^{E}(k)$ is satisfied and therefore we have $a^{u} \sim a^{B} \sim a^{E}=-7 / 2$. The similarity forms (4.7), (4.15), and (4.16) imply that the characteristic decay times of $Q^{u}(k), Q^{B}(k), G^{u}(k)$ and $G^{B}(k)$ are of the order of $\left(B_{0} k\right)^{-1}$. Hence, the assumptions (D1) and (D2) of the present analysis are consistent with the results.

The results of this section are summarized as

$$
\begin{aligned}
& Q^{E}(k, t, s)=\frac{A}{2 \pi} \epsilon^{1 / 2} B_{0}^{1 / 2} k^{-7 / 2} g\left(B_{0} k(t-s)\right), \\
& Q^{R}(k, t, s)=\frac{A^{R}}{2 \pi} \epsilon B_{0}^{-1} k^{-4} r^{R}\left(B_{0} k(t-s)\right), \\
& G^{u}(k, t, s)=G^{B}(k, t, s)=g\left(B_{0} k(t-s)\right),
\end{aligned}
$$

for $k \gg k_{B}$ in the inertial subrange, where $g$ is given explicitly in (4.8) and $r^{R}$ is the solution of (4.18) with $r^{R}(0)=1$. Note that the form of $G^{u}$ and $G^{B}$ in (4.39) is exactly the same as that of $G^{u}$ in DIA ${ }^{25}$ for hydrodynamic turbulence when $B_{0}$ is replaced by $U:=\sqrt{\left\langle u_{i}^{2}\right\rangle}$. Equations (4.37) and (4.38) with (4.11) and (4.12) imply

$$
\begin{aligned}
& E(k, t)=2 A \epsilon^{1 / 2} B_{0}^{1 / 2} k^{-3 / 2}, \\
& E^{R}(k, t)=A^{R} \epsilon B_{0}^{-1} k^{-2},
\end{aligned}
$$

which may be rewritten as

$$
\begin{aligned}
& E^{u}(k, t)=\epsilon^{1 / 2} B_{0}^{1 / 2} k^{-3 / 2}\left[A+\frac{A^{R}}{2} \Delta(k)\right], \\
& E^{B}(k, t)=\epsilon^{1 / 2} B_{0}^{1 / 2} k^{-3 / 2}\left[A-\frac{A^{R}}{2} \Delta(k)\right] .
\end{aligned}
$$

For sufficiently high $k$ such that $\Delta(k) \ll 1$, (4.42) and (4.43) are consistent with the IK spectrum, $E^{u}(k, t)=E^{B}(k, t)$ $=A \epsilon^{1 / 2} B_{0}^{1 / 2} k^{-3 / 2}$. The scaling $E^{R}(k, t) \propto k^{-2}$ in (4.41) is consistent with that obtained in the analysis of the EDQNM approximation in Ref. 4.

\section{NUMERICAL ESTIMATES}

From (4.8), one obtains

$$
\int_{0}^{\infty} d \tau g(\tau)=1, \quad \int_{0}^{\infty} d \tau[g(\tau)]^{2} \doteq 0.849,
$$

where the former is the exact result, and $\doteq$ denotes a numerical estimate. The function $r^{R}$ is obtained by solving (4.18) numerically and plotted in Fig. 1. An iterative method that is similar to that in Ref. 26 is used in the numerical calculation. We obtain from the numerical solution of $r^{R}$ that

$$
\int_{0}^{\infty} d \tau r^{R}(\tau) \doteq 0.495, \quad I_{G R} \doteq 0.500 .
$$

One can estimate that $K \doteq 1.92$ from (4.25). Then (4.23) implies

$$
A \doteq 0.722 .
$$

From (4.30), we obtain the numerical estimate $K_{1}$ $\doteq-0.1172$. By using (4.33), (5.2), and (5.3), the estimate results in

$$
A^{R} \doteq-0.384 \text {. }
$$

Now, the functions $W(\alpha), \nu^{u u}\left(k_{c}\right), \nu^{B B}\left(k_{c}\right), \nu^{u}\left(k, k_{c}\right)$, and $\nu^{B}\left(k, k_{c}\right)$ can be completely determined, respectively, by (4.27), (3.26), (3.27), (3.29), and (3.30) with the substitution of the similarity forms of $Q$ and $G$ given by (4.37)-(4.39) 


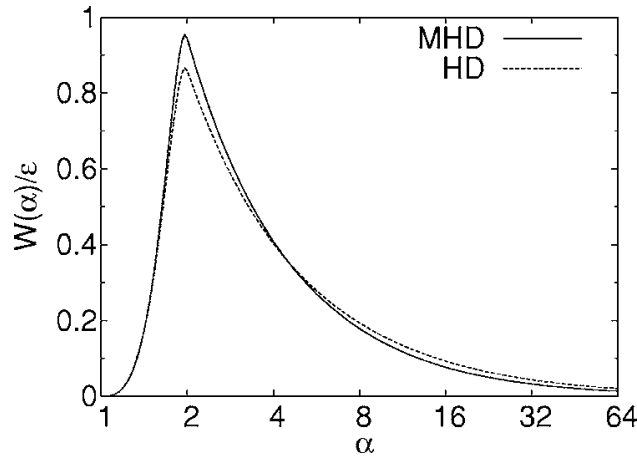

FIG. 2. Function $W(\alpha) / \epsilon$ for the MHD turbulence. The function for the hydrodynamic (HD) case is also plotted for reference.

with (5.3) and (5.4). Since we are interested in the asymptotic forms of the functions for sufficiently high $k$, we will neglect the terms of $O[\Delta(k)]$ in the following. This is formally equivalent to putting $Q^{R}(k)=0$.

The function $W(\alpha)$ normalized by $\epsilon$ is plotted in Fig. 2 . The corresponding function in the hydrodynamic case is also plotted for reference (cf. Fig. 2 of Ref. 24). One can see from the figure that the value of $W(\alpha)$ in MHD turbulence excesses slightly that in the hydrodynamic turbulence near the peak $\alpha \simeq 2$. This implies that the triad interactions that contribute to the total energy flux in the MHD turbulence are somewhat more local in wave-vector space than those in the hydrodynamic turbulence.

The estimates of eddy viscosity $\nu^{u u}\left(k_{c}\right)$ and eddy magnetic diffusivity $\nu^{B B}\left(k_{c}\right)$ are given, respectively, as

$$
\begin{aligned}
& \nu^{u u}\left(k_{c}\right) \doteq 0.38 \epsilon^{1 / 2} B_{0}^{-1 / 2} k_{c}^{-3 / 2}, \\
& \nu^{B B}\left(k_{c}\right) \doteq 0.27 \epsilon^{1 / 2} B_{0}^{-1 / 2} k_{c}^{-3 / 2} .
\end{aligned}
$$

It is found that $\nu^{u u}\left(k_{c}\right)>\nu^{B B}\left(k_{c}\right)$, which implies that the kinetic energy at low wave numbers $\left(\ll k_{c}\right)$ transfers more efficiently than the magnetic counterpart does to the region of wave numbers larger than $k_{c}$ within the inertial subrange. Note that each of kinetic and magnetic energy at low wave numbers may transfer to either of kinetic and magnetic energy in the region of wave numbers larger than $k_{c}$ since kinetic energy $E^{u}$ and magnetic energy $E^{B}$ may interchange during the transfer process while the total energy $E=E^{u}$ $+E^{B}$ conserves.

The estimates of $k$-dependent eddy viscosity $\nu^{u}\left(k, k_{c}\right)$ and eddy magnetic diffusivity $\nu^{B}\left(k, k_{c}\right)$ are given, respectively, as

$$
\begin{aligned}
& \nu^{u}\left(k, k_{c}\right)=\epsilon^{1 / 2} B_{0}^{-1 / 2} k_{c}^{-3 / 2} f^{u}\left(\frac{k}{k_{c}}\right), \\
& \nu^{B}\left(k, k_{c}\right)=\epsilon^{1 / 2} B_{0}^{-1 / 2} k_{c}^{-3 / 2} f^{B}\left(\frac{k}{k_{c}}\right),
\end{aligned}
$$

where $f^{u}$ and $f^{B}$ are functions plotted in Fig. 3. The figure shows that $\nu^{u}\left(k, k_{c}\right)$ is larger than $\nu^{B}\left(k, k_{c}\right)$ in the entire wavenumber range, $0<k<k_{c}$, where $\nu^{u}\left(k, k_{c}\right)$ and $\nu^{B}\left(k, k_{c}\right)$ are defined.

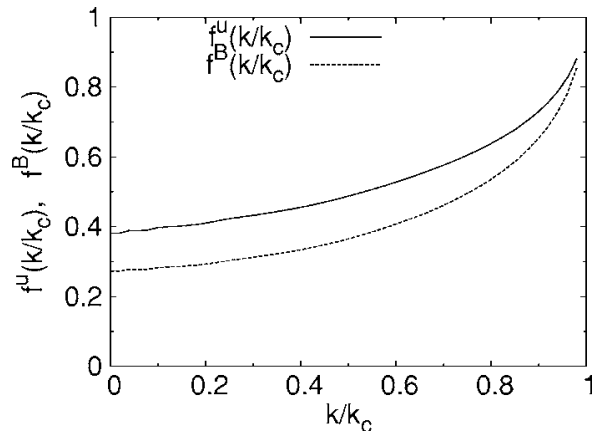

FIG. 3. Rescaled eddy viscosity $f^{u}\left(k, k_{c}\right)$ and eddy magnetic diffusivity $f^{B}\left(k, k_{c}\right)$ as functions of $k / k_{c}$.

\section{DNS}

\section{A. Setup}

In order to verify the universal energy spectra (4.40) and (4.41) with (5.3) and (5.4), we performed DNS of a forced quasi-isotropic MHD turbulence. Periodic boundary conditions are applied with period $2 \pi$ in each of the Cartesian coordinates and an alias-free spectral method is used. The fourth-order Runge-Kutta method is used for the time marching.

Let $X_{i}^{\alpha}(\mathbf{k}, t)\left[\alpha=u\right.$ or $B, i=1,2$, or $3, \mathbf{k}=\left(k_{1}, k_{2}, k_{3}\right), t$ $=m \Delta t]$ be the simulated velocity and magnetic field in the Fourier space, where $\left(k_{1}, k_{2}, k_{3}\right)$ is a set of integers satisfying $|\mathbf{k}|=k_{1}^{2}+k_{2}^{2}+k_{3}^{2}<k_{\max }, k_{\max }$ is the maximum resolved wave number, $m$ is 0 or a positive integer, and $\Delta t$ is the minimum increment of time step. The kinetic energy $E^{u}(t)$, magnetic energy $E^{B}(t)$, cross helicity $E^{C}(t)$, magnetic helicity $H^{M}(t)$, kinetic energy dissipation rate $\epsilon^{u}(t)$, and magnetic energy dissipation rate $\epsilon^{B}(t)$ per unit mass at time $t$ are given, respectively, by

$$
\begin{aligned}
& E^{u}(t)=\frac{1}{2} \sum_{\mathbf{k}} \widetilde{Q}_{i i}^{u u}(\mathbf{k}, t), \quad E^{B}(t)=\frac{1}{2} \sum_{\mathbf{k}} \widetilde{Q}_{i i}^{B B}(\mathbf{k}, t), \\
& E^{C}(t)=\sum_{\mathbf{k}} \widetilde{Q}_{i i}^{u B}(\mathbf{k}, t), \quad H^{M}(t)=\sum_{\mathbf{k}} i \epsilon_{j m n} \frac{k_{j}}{k^{2}} \widetilde{Q}_{m n}^{B B}(\mathbf{k}, t), \\
& \epsilon^{u}(t)=\nu^{u} \sum_{\mathbf{k}} k^{2} \widetilde{Q}_{i i}^{u u}(\mathbf{k}, t), \quad \epsilon^{B}(t)=\nu^{B} \sum_{\mathbf{k}} k^{2} \widetilde{Q}_{i i}^{B B}(\mathbf{k}, t),
\end{aligned}
$$

where

$$
\widetilde{Q}_{i j}^{\alpha \beta}(\mathbf{k}, t):=X_{i}^{\alpha}(\mathbf{k}, t) X_{j}^{\beta}(-\mathbf{k}, t),
$$

and $\epsilon_{i j k}$ is the third-order antisymmetric tensor with $\epsilon_{123}=1$. The spectra associated with $E^{u}(t), E^{B}(t), E^{C}(t)$, and $H^{M}(t)$ are given, respectively, by

$$
E^{u}(k, t)=\frac{1}{2} \sum_{k^{\prime}=k} \widetilde{Q}_{i i}^{u u}\left(\mathbf{k}^{\prime}, t\right),
$$




$$
\begin{aligned}
& E^{B}(k, t)=\frac{1}{2} \sum_{k^{\prime}=k} \widetilde{Q}_{i i}^{B B}\left(\mathbf{k}^{\prime}, t\right), \\
& E^{C}(k, t)=\sum_{k^{\prime}=k} \widetilde{Q}_{i i}^{u B}\left(\mathbf{k}^{\prime}, t\right), \\
& E^{H}(k, t)=\sum_{k^{\prime}=k} i \epsilon_{j m n} \frac{k_{j}}{k} \widetilde{Q}_{m n}^{B B}\left(\mathbf{k}^{\prime}, t\right),
\end{aligned}
$$

where $k=1, \ldots, k_{\max }$ and $\Sigma_{k^{\prime}=k}$ denotes the summation with respect to $\mathbf{k}^{\prime}$ over the shell $k-1 / 2<\left|\mathbf{k}^{\prime}\right| \leq k+1 / 2$. The total energy flux $\Pi(k, t)$ flowing into wave numbers larger than $k$ is given by

$$
\begin{aligned}
\Pi(k, t)= & \frac{1}{2} \sum_{k^{\prime}>k}\left[\left(\frac{\partial u_{i}\left(\mathbf{k}^{\prime}, t\right)}{\partial t}\right)_{\nu=\lambda=0} u_{i}\left(-\mathbf{k}^{\prime}, t\right)\right. \\
& \left.+\left(\frac{\partial B_{i}\left(\mathbf{k}^{\prime}, t\right)}{\partial t}\right)_{\nu=\lambda=0} B_{i}\left(-\mathbf{k}^{\prime}, t\right)\right],
\end{aligned}
$$

where the time derivatives are computed by neglecting dissipation terms.

In the present DNS, random external forces $\mathbf{f}^{u}(\mathbf{k}, t)$ and $\mathbf{f}^{B}(\mathbf{k}, t)$ are applied to $\mathbf{u}(\mathbf{k}, t)$ and $\mathbf{B}(\mathbf{k}, t)$, respectively, in a low-wave-number range in order to achieve statistical quasistationarity in a high-wave-number range. For $k \leq k_{f}$, $\mathbf{f}^{u}(\mathbf{k}, t)$ and $\mathbf{f}^{B}(\mathbf{k}, t)$ satisfy $\left\langle\mathbf{f}^{\alpha}(\mathbf{k}, t)\right\rangle=\mathbf{0}$,

$$
\begin{aligned}
& \left\langle f_{i}^{\alpha}(\mathbf{k}, t+\tau) f_{j}^{\alpha}\left(-\mathbf{k}^{\prime}, t\right)\right\rangle=F^{\alpha} P_{i j}(\mathbf{k}) \delta_{\mathbf{k}, \mathbf{k}^{\prime}} e^{-\mid \tau / T_{f}}, \\
& \left\langle f_{i}^{u}(\mathbf{k}, t+\tau) f_{j}^{B}\left(-\mathbf{k}^{\prime}, t\right)\right\rangle=0 \\
& \boldsymbol{\epsilon}_{j m n} k_{j}\left\langle f_{m}^{B}(\mathbf{k}, t+\tau) f_{n}^{B}\left(-\mathbf{k}^{\prime}, t\right)\right\rangle=0
\end{aligned}
$$

where $\langle\cdot\rangle$ denotes a random average (see the Appendix for the method used to generate such random forces). For $k$ $>k_{f}$, the forcings are not applied, i.e., $\mathbf{f}^{\alpha}(\mathbf{k}, t)=\mathbf{0}$. Equations (6.11) and (6.12) imply that $\mathbf{f}^{u}$ and $\mathbf{f}^{B}$ do not inject cross helicity $E^{C}$ and magnetic helicity $H^{M}$ into the system on average. We set $F^{u}=F^{B}=F$, i.e., kinetic energy and magnetic energy are injected at the same rate. The correlation time $T_{f}$ of the external forces is set to $T_{f}=2.0$, which is of the order of the large-eddy turnover time $L^{u} / u_{0}$, where $u_{0}$ $:=\left(\overline{|\mathbf{u}|^{2}} / 3\right)^{1 / 2}=\left(2 E^{u} / 3\right)^{1 / 2}$ is the typical amplitude of the velocity fluctuation with $\bar{X}$ denoting the spatial average of a quantity $X$, and $L^{u}:=\left(\pi / 2 u_{0}^{2}\right) \int_{0}^{k_{\max }} d k k^{-1} E^{u}(k)$ is the integral length scale. We set $\nu=\lambda$ and the value of $\nu$ is chosen to satisfy $k_{\max } \eta_{I K} \simeq 2$, where $\eta_{I K}:=\left(\nu^{2} B_{0} / \epsilon\right)^{1 / 3}$ is IK microscale, $B_{0}:=\left(|\mathbf{B}|^{2} / 3\right)^{1 / 2}=\left(2 E^{B} / 3\right)^{1 / 2}$ is the typical amplitude of the magnetic field fluctuation, and $\epsilon:=\epsilon^{u}+\epsilon^{B}$ is the total energy dissipation rate.

TABLE I. Basic parameters of DNS.

\begin{tabular}{lcclcccc}
\hline \hline & $N$ & $k_{\max }$ & $\nu\left(\times 10^{-3}\right)$ & $\Delta t\left(\times 10^{-3}\right)$ & $F\left(\times 10^{-3}\right)$ & $k_{f}$ & $T_{f}$ \\
\hline RUN256 & 256 & 120 & $0.6 / 1.3^{\mathrm{a}}$ & 4.0 & 0.9 & 2.5 & 2.0 \\
RUN512 & 512 & 241 & 0.45 & 1.0 & 0.9 & 2.5 & 2.0 \\
\hline
\end{tabular}

The value of $\nu$ in RUN256 is 0.6 for $t=0.0-4.0$ and 1.3 for $t=4.0-8.0$.
TABLE II. Characteristic quantities of the final states of RUN256 $(t=8.0)$ and RUN512 $(t=2.8)$.

\begin{tabular}{lcccccccc}
\hline \hline & $E^{u}$ & $E^{B}$ & $B_{0}$ & $\epsilon$ & $\epsilon^{u}$ & $\epsilon^{B}$ & $E^{C}$ & $H^{M}\left(\times 10^{-3}\right)$ \\
\hline RUN256 & 0.578 & 0.893 & 0.772 & 0.260 & 0.105 & 0.155 & 0.0530 & 0.789 \\
RUN512 & 0.574 & 1.08 & 0.848 & 0.260 & 0.109 & 0.150 & 0.0597 & 38.3 \\
\hline \hline
\end{tabular}

\section{B. Results}

We performed two runs, RUN256 and RUN512. Basic parameters of the runs are listed in Table $\mathrm{I}$, where $N$ is the number of grid points along each Cartesian axis. The initial fields of $\mathbf{u}$ and $\mathbf{B}$ for RUN256 are generated randomly under the constraints

$$
\begin{aligned}
& E^{u}(k, 0) \simeq E^{B}(k, 0) \simeq C k^{4} \exp \left(-2 \frac{k^{2}}{k_{p}^{2}}\right), \\
& E^{C}(k, 0) \simeq E^{H}(k, 0) \simeq 0,
\end{aligned}
$$

where we put $k_{p}=2$ and the constant $C$ is determined to satisfy $E^{u}(0) \simeq E^{B}(0) \simeq 0.5$. RUN256 was performed up to $t$ $=8.0$, at which $\epsilon^{u}$ and $\epsilon^{B}$, the quantities characterizing the behavior of small scales, have already been in a quasistationary state (figure omitted). The final state $(t=8.0)$ of RUN256 was used as the initial condition of RUN512. RUN512 was performed up to $t=2.8$. The total energy dissipation rate $\epsilon$ reaches its peak value at $t \simeq 2.3$ and changes moderately afterward (figure omitted).

We expect that the small scales in the final states of RUN256 and RUN512 represent statistically quasistationary states of MHD turbulence. Characteristics of turbulence at the final states are given in Tables II and III. Here, $L^{B}$ $:=\left(\pi / 2 B_{0}^{2}\right) \int_{0}^{k_{\max }} d k k^{-1} E^{B}(k)$ is the integral scale associated with $\mathbf{B}, \lambda^{u}:=\left(15 \nu^{u} u_{0}^{2} / \epsilon^{u}\right)^{1 / 2}$ and $\lambda^{B}:=\left(15 \nu^{B} B_{0}^{2} / \epsilon^{B}\right)^{1 / 2}$ are, respectively, the Taylor microscales associated with $\mathbf{u}$ and B, and $\eta:=\left(\nu^{3} / \epsilon^{u}\right)^{1 / 4}$ is the Kolmogorov microscale. $R_{\lambda}^{u}:=u_{0} \lambda^{u} / \nu^{u}=\left(20 / 3 \epsilon^{u} \nu^{u}\right)^{1 / 2} E^{u} \quad$ and $\quad R_{\lambda}^{B}:=B_{0} \lambda^{B} / \nu^{B}$ $=\left(20 / 3 \epsilon^{B} \nu^{B}\right)^{1 / 2} E^{B}$ are the Taylor microscales Reynolds numbers associated with $\mathbf{u}$ and $\mathbf{B}$, respectively, and $R_{\lambda}^{M}$ $:=\left(20 E^{u} E^{B} / 3 \epsilon \nu\right)^{1 / 2}$ is the Taylor microscale Reynolds number for MHD turbulence introduced in Ref. 12. All results given in the following are those of the final states.

Figure 4 shows the total energy flux $\Pi(k)$ normalized by $\epsilon$. Note that $\Pi(k) \sim \epsilon$ should be satisfied in the inertial subrange. One can see from the figure that the upper bound of the inertial subrange is $k \simeq 0.1 \eta_{I K}^{-1}$. Considering that $k_{f}$ is smaller than the lower-boundary wave number of the inertial subrange, one may conclude that RUN512 has quite a narrow inertial subrange (less than one decade) and that

TABLE III. Length scales and Reynolds numbers of the final states of RUN256 $(t=8.0)$ and RUN512 $(t=2.8)$.

\begin{tabular}{cccccccccc}
\hline \hline & $L^{u}$ & $L^{B}$ & $\lambda^{u}$ & $\lambda^{B}$ & $\eta\left(\times 10^{-3}\right)$ & $\eta_{I K}\left(\times 10^{-3}\right)$ & $R_{\lambda}^{u}$ & $R_{\lambda}^{B}$ & $R_{\lambda}^{M}$ \\
\hline RUN256 & 1.31 & 1.10 & 0.267 & 0.274 & 12.0 & 17.1 & 128 & 162 & 101 \\
RUN512 & 1.19 & 1.32 & 0.154 & 0.180 & 5.38 & 8.71 & 211 & 339 & 188 \\
\hline \hline
\end{tabular}




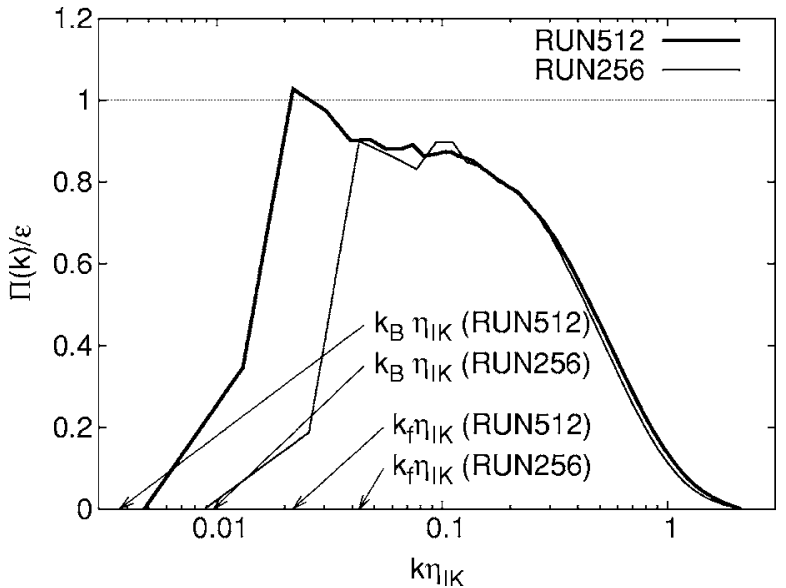

FIG. 4. Total energy flux $\Pi(k)$ normalized by the total energy dissipation rate $\epsilon$.

RUN256 has almost none. In the following, the data from RUN512 are used to examine the spectra in the inertial subrange, and the data from RUN256 are shown only for reference. We call the wave-number range $0.03 \eta_{I K}^{-1}<k \leq 0.1 \eta_{I K}^{-1}$ in RUN512 "inertial subrange" for the sake of convenience. Note that $k_{B}$ is smaller than the lower-boundary wave number of the "inertial subrange" (see Fig. 4), which implies that $\Delta(k)<1$ is satisfied in the entire "inertial subrange."

The spectra $E^{u}(k), E^{B}(k), E^{C}(k)$, and $E^{H}(k)$ of RUN512 are shown in Fig. 5. The spectra $E^{C}(k)$ and $E^{H}(k)$ decease with respect to $k$ much faster than $E^{u}(k)$ and $E^{B}(k)$. The values of $E^{C}(k)$ and $E^{H}(k)$ are typically about 10 times smaller than those of $E^{u}(k)$ and $E^{B}(k)$ in the "inertial subrange." This is consistent with the assumption (3.1)-(3.3) of isotropy. There is a systematic deviation from the equipartition $E^{u}(k)=E^{B}(k)$ throughout the entire wave-number range. The IK spectrum $E^{u}(k)=E^{B}(k)=A \epsilon^{1 / 2} k^{-3 / 2}$ with the LRA value of $A[(5.3)]$ is also plotted in the figure. The spectrum $E^{u}(k)$ in the "inertial subrange" has a smaller amplitude and a slightly more gentle slope than the IK spectrum with (5.3).

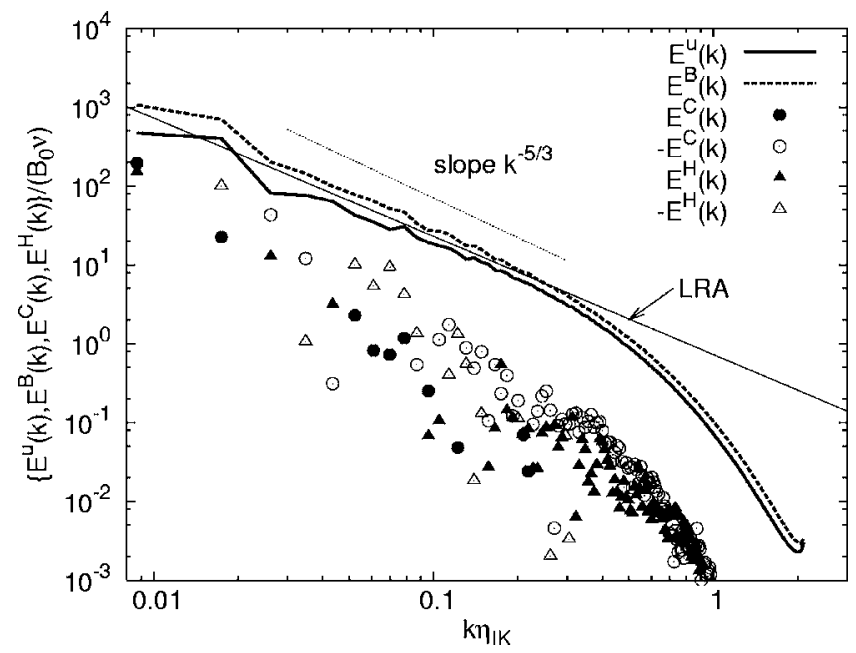

FIG. 5. Energy spectra $E^{u}(k), E^{B}(k) E^{C}(k)$, and $E^{H}(k)$ of RUN512. IK spectrum, $E^{u}(k)=E^{B}(k)=A B_{0}^{1 / 2} \epsilon^{1 / 2} k^{-3 / 2}$, with the LRA estimate, $A=0.722$, is given in line. The slope $k^{-5 / 3}$ is shown for reference.

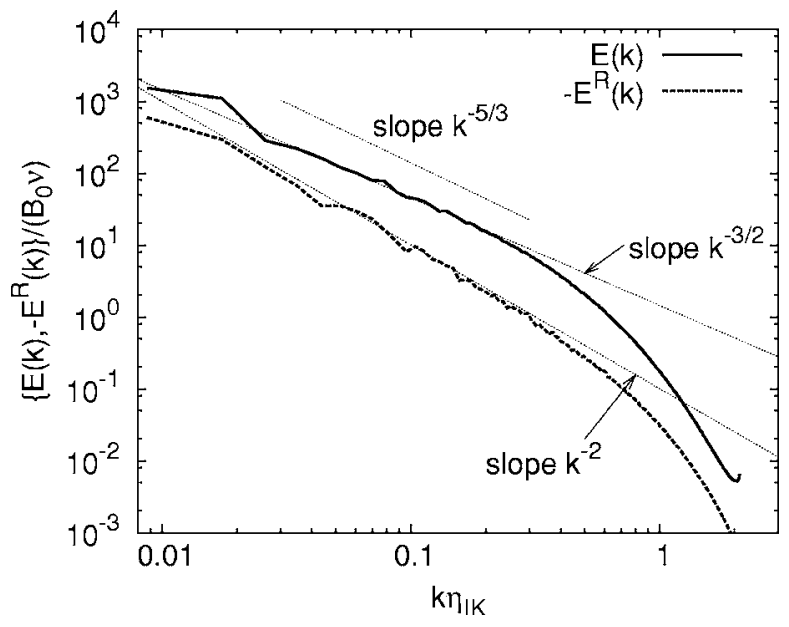

FIG. 6. Total energy spectrum $E(k)$ and residual energy spectrum $E^{R}(k)$ of RUN512. The slopes $k^{-3 / 2}$ and $k^{-2}$ of the LRA prediction are given in lines. The slope $k^{-5 / 3}$ is shown for reference.

On the other hand, $E^{B}(k)$ has a larger amplitude and a slightly steeper slope than the IK spectrum with (5.3).

The total energy spectrum $E(k)$ and the residual energy spectrum $E^{R}(k)$ of RUN512 are given in Fig. 6. It is found that $E(k)$ in RUN512 is consistent with the IK scaling $k^{-3 / 2}$ predicted by LRA. Furthermore, $E^{R}(k)$ in RUN512 is consistent with the prediction of the LRA regarding the scaling $k^{-2}$ and the sign $E^{R}(k)<0$. The scalings of the spectra $E(k)$ $\propto k^{-3 / 2}$ and $E^{R}(k) \propto k^{-2}$ shown in LRA and the present DNS suggest that the relative deviation from the equipartition $e^{R}(k):=\left|E^{R}(k)\right| / E(k)$ decreases with respect to $k$ like $\Delta(k)$ $\propto k^{-1 / 2}$ and that the equipartition $E^{u}(k)=E^{B}(k)$ may be asymptotically realized in the universal wave-number range, i.e., the sufficiently high-wave-number range in MHD turbulence at very high Reynolds numbers. The deviation of $E^{u}(k)$ and $E^{B}(k)$ from the equipartition in the present DNS can be explained by the narrowness of the "inertial subrange," i.e., $\Delta(k)=\epsilon B_{0}^{-3 / 2} k^{-1 / 2}$ is not small enough in the "inertial subrange." The decrease of $e^{R}(k)$ is similar to that of anisotropy in hydrodynamic turbulences (see, e.g., Ref. 27 and references therein) in the sense that both $e^{R}(k)$ and anisotropy decrease with the increase of $k$ and tend to vanish in the universal scale range, however they remain for finite wave number in the turbulence at a finite Reynolds number.

For a close examination of the scaling exponent of $E(k)$ and the universal constant $A$, the compensated spectrum $k^{3 / 2} E(k) /\left(B_{0}^{1 / 2} \epsilon^{1 / 2}\right)$ is plotted in Fig. 7, together with the compensated spectra associated with $E^{u}(k)$ and $E^{B}(k)$. The slope of $k^{-1 / 6}$ that corresponds to $E(k) \propto k^{-5 / 3}$ is also given in the figure for reference. The compensated spectrum associated with $E(k)$ of RUN512 is almost constant in the "inertial subrange" and thus it is evident that the scaling of $E(k)$ is closer to $k^{-3 / 2}$ than to $k^{-5 / 3}$. The constant $2 A$ estimated by averaging the compensated spectrum associated with $E(k)$ in the "inertial subrange" is

$$
2 A=1.47 \pm 0.09 \text {, }
$$

where \pm 0.09 is the standard deviation. The estimate is in good agreement with the LRA estimate $2 A \doteq 1.44$ from (5.3). 


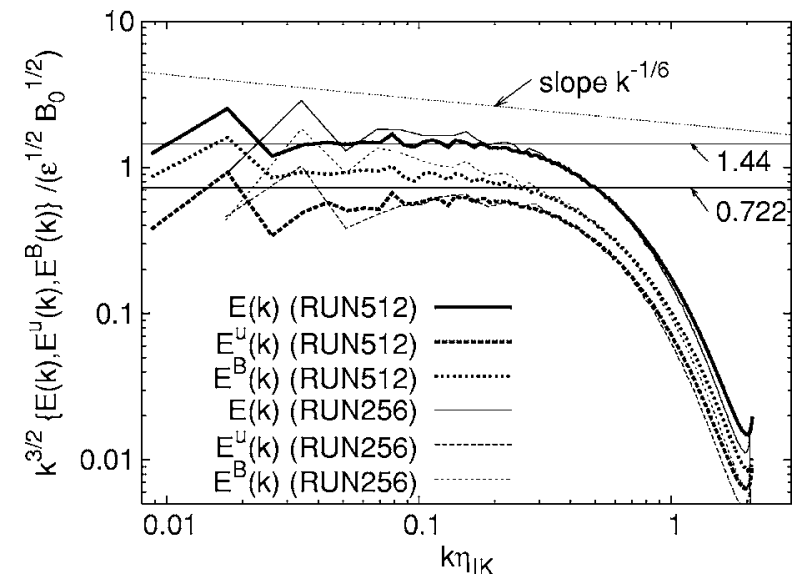

FIG. 7. Compensated energy spectra $k^{3 / 2}\left\{E(k), E^{u}(k), E^{B}(k)\right\} /\left(B_{0}^{1 / 2} \epsilon^{1 / 2}\right)$. $A$ $\doteq 0.722$ and $2 A \doteq 1.44$ are the LRA estimates. The slope $k^{-1 / 6}$ corresponds to $E(k) \propto k^{-5 / 3}$

The compensated spectra associated with $E(k), E^{u}(k)$ and $E^{B}(k)$ of RUN256 are also plotted in the figure for reference. One can observe a tendency that $E(k)$ approaches the universal spectrum (4.40) and $E^{u}(k)$ and $E^{B}(k)$ approach the equipartition with the increase of the Reynolds number.

The compensated residual energy spectrum $-k^{2} E^{R}(k) /\left(\epsilon B_{0}^{-1}\right)$ is plotted in Fig. 8. The spectrum of RUN512 is nearly constant in the "inertial subrange," which implies that $E^{R}(k) \propto k^{-2}$. The constant $A^{R}$ estimated by averaging the compensated spectrum over the "inertial subrange" is

$$
A^{R}=-1.49 \pm 0.25,
$$

where \pm 0.25 is the standard deviation. The estimate from the DNS is about four times larger in its magnitude than the LRA estimate (5.4).

Possible sources of the discrepancy between the values of $A^{R}$ in LRA and the DNS are (i) the insufficient width of the "inertial subrange" in the DNS, and (ii) the inadequacy of LRA.

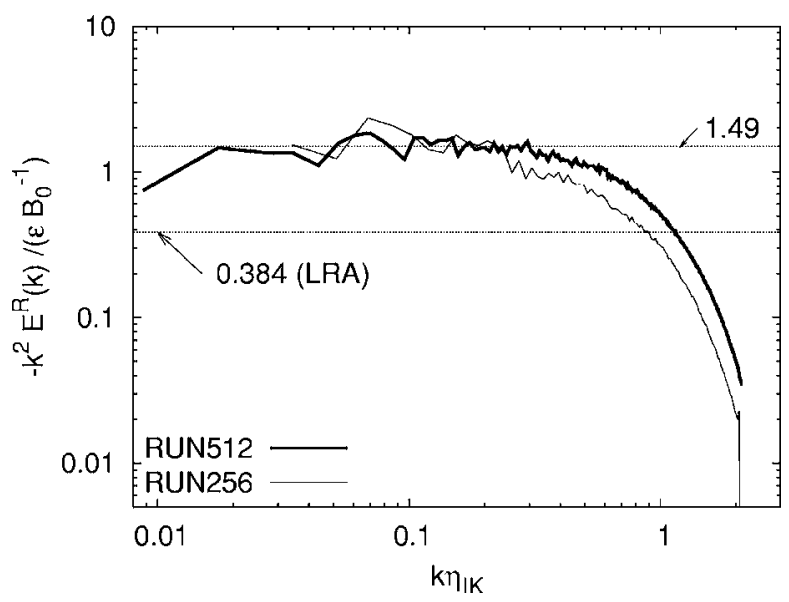

FIG. 8. Compensated residual energy spectrum $-k^{2} E^{R}(k) /\left(\epsilon B_{0}^{-1}\right) . \quad A^{R}$ $\doteq-0.384$ is the LRA estimate and $A^{R} \simeq-1.49$ is obtained from the fit of the DNS data of RUN512.
Regarding (i), note that the estimates based on LRA in Sec. V are obtained in the limit of very large width of the inertial subrange, i.e., very high Reynolds number. There is a possibility that the convergence of the value of $A^{R}$ with the increase of Reynolds number is much slower than that of $A$, and that the estimate $A^{R} \simeq-1.49$ in RUN512 does not approximate the limiting value. Comparison between the spectra of RUN256 and RUN512 gives a partial support to this scenario. One can observe from Figs. 7 and 8 that the difference in the compensated spectrum associated with $E^{R}(k)$ between RUN256 and RUN512 is much larger than that in the compensated spectrum associated with $E(k)$. In the present setting of DNS, the magnitude of the compensated spectrum associated with $E^{R}(k)$ decreases in the "inertial subrange" and increases in the dissipation range with the increase of the Reynolds number. It is desirable to perform DNS with a larger number of grid points in order to examine the dependence of $E^{R}(k)$ on the Reynolds number in the higher Reynolds number regime.

Regarding (ii), the quality of closure approximations depends on the choice of the variables in terms of which the renormalized expansions are constructed. The variables are called representatives in Ref. 14. The estimate of $A^{R}$ may be improved with some alternative choice of representatives. A candidate for such representatives are two-point correlation functions and two-point response functions associated with the variables measured in the coordinates moving with $\mathbf{u} \pm \mathbf{B}$, i.e., moving, relative to the fluid, in the parallel and antiparallel direction of the local magnetic field with the local Alfvén velocity. Such variables were introduced in Ref. 3. However, to the authors' knowledge, the closure analysis that corresponds to the present study based on these variables has not been done yet and this is left for a future challenge.

\section{DISCUSSION}

Within IK phenomenology, the Alfvén waves with wave numbers in the inertial subrange are propagating at speed of the order of $B_{0}$ in the coordinate system moving with the local velocity of fluid, while the coherence length of each wave is of the order of its own wavelength due to the strong nonlinearity. Consequently, the time scale of the decorrelation associated with two-time Lagrangian correlation functions is of the order of $\left(B_{0} k\right)^{-1}$. This result is derived within the framework of LRA by itself, as was shown in Sec. IV. The total energy spectrum $E(k)$ obtained by the present DNS is in good agreement with LRA not only in its scaling $k^{-3 / 2}$ but also in its universal constant $A$ as shown in Sec. VI. This suggests that the turbulence in the present DNS is a strong turbulence and that LRA provides an appropriate theoretical framework for strong MHD turbulence, as well as for purely hydrodynamic turbulence.

The present situation is quite different from that for weak turbulence. ${ }^{11}$ Within the theory of weak turbulence, it is assumed that the nonlinearity is weak and that the coherence length of each wave is much larger than its own wavelength. Therefore, the decorrelation time of the two-time Lagrangian correlation functions is much larger than $\left(B_{0} k\right)^{-1}$. 
The time scale $\left(B_{0} k\right)^{-1}$ provides the order of the inverse of the frequency of the wave with wave number $k$.

It is known that the so-called "bump" of the energy spectrum is observed in the near-dissipation range in DNS of the hydrodynamic turbulence (see, e.g., Ref. 28). Although there is a possibility that the scaling $E(k) \propto k^{-3 / 2}$ in the present DNS is a spurious effect of the "bump, ${ }^{29}$ we draw a scenario that the 'bump' is negligibly small, if it exists, in the isotropic incompressible MHD turbulence and that $E(k)$ $\propto k^{-3 / 2}$ is the universal scaling, since $E(k)$ in the DNS agrees with that in LRA not only with respect to the scaling exponent but also to the universal constant $A$. The "bump" in MHD turbulence should be investigated further by using DNS with higher Reynolds numbers, which is beyond the scope of the present study.

The scaling of $E(k) \propto k^{-3 / 2}$ found in the present DNS is different from $E(k) \propto k^{-5 / 3}$ observed in DNSs by BM and MG for the decaying quasi-isotropic MHD turbulence. The discrepancy may be attributed to the difference of the regimes of wave number in which DNSs are performed. The analysis in Sec. IV suggests that $E(k) \propto k^{-3 / 2}$ and $E^{u}(k) \sim E^{B}(k)$ are satisfied in the wave-number regime $k \gg k_{B}$ within the inertial subrange where $k_{B}=\epsilon B_{0}^{-3}$. The scaling of $E(k)$ may be different and $E^{u}(k) \sim E^{B}(k)$ may not hold in the wave-number regime $k \leq k_{B}$. Let $k_{0}$ be the bottom wave number of the scaling ranges in the DNSs. In the present DNS, $k_{0} / k_{B}>1$ is satisfied as shown in Fig. 4. The values of $\epsilon$ and $B_{0}$ are not given explicitly and thus the values of $k_{B}$ are not available for DNSs by MG and BM. Let us examine the relative deviation from the equipartition $e^{R}(k)=\left|E^{R}(k)\right| / E(k)$ in the DNSs instead of $k_{B}$. The relative deviation $e^{R}(k)$ in DNSs both of MG and of the present study decreases with respect to $k$ (see Fig. 1 in MG and Fig. 6 in this paper. The corresponding figure is not given in $\mathrm{BM}$ ). The deviation $e^{R}\left(k_{0}\right) \simeq 0.3$ in RUN512 of the present study is smaller than $e^{R}\left(k_{0}\right) \simeq 0.7$ in MG. Hence, we conjecture that $k_{0} / k_{B}$ in RUN512 is larger than that in MG, which implies that the range of wave number simulated in RUN512 is nearer to the universal regime of wave number, i.e., the sufficiently high-wave-number range in MHD turbulence at very high Reynolds numbers, in comparison with that in MG.

In performing DNS of the universal regime of wave number in MHD turbulence, we omitted the simulation of the lower wave numbers outside of the universal regime due to the limitation of the computational resource. The random force applied, in the present DNS, at low wave numbers within the range of the simulation may be interpreted as a model of energy transfer from the omitted lower wave numbers to those in the range of the simulation. Hence, it is reasonable for us to set the scale of correlation time for the forcing to the order of the turnover time of the largest eddy within the range of the simulation. We set the ratio of injection by the forcing for $E^{u}$ to that for $E^{B}$ to unity in order to keep $e^{R}(k)$ small in the low-wave-number range where the forcing is applied. This is because, in decaying MHD turbulence by MG, $e^{R}(k)$ is small for high $k$, say higher than $k^{*}$, and because the target of the present DNS is the range of wave number "higher" than $k^{*}$ for turbulence at a "higher" Reynolds number.
One of the candidates of the physical situation that corresponds to the present analysis would be sufficiently small scales in the solar wind turbulence. Note that, for the purely hydrodynamic case, wind tunnel turbulence may be regarded as a forced quasistationary turbulence when the region of the measurement is fixed in the laboratory system, since turbulent fluctuation generated in the upwind region is continuously flowing into the region of the measurement. It is well accepted that the statistics of wind tunnel turbulence far from the wall is almost isotropic at small scales when the Reynolds number is high. Turbulence in the solar wind measured at a fixed distance from the Sun can be the MHD analog of the wind tunnel turbulence. Hence, the forced quasi-isotropic MHD turbulence can be an appropriate approximation for the small scales of the turbulence in the solar wind. Note that a quasi- $k^{-3 / 2}$ spectrum is obtained in the high-frequency region (i.e., at small scales) of the observation of the solar wind turbulence (e.g., see Fig. 1 in Ref. 30). The solar wind turbulence is actually anisotropic in the large scales. Quantitative estimates on the effect of the anisotropy on the statistics of the small scales is an interesting problem.

The present analysis using LRA equations (3.7)-(3.10) is restricted to the isotropic MHD turbulence. In MHD turbulence with a finite Reynolds number, components of the spectrum tensor $Q_{i j}^{\alpha \beta}(\mathbf{k})$, which are related to cross helicity $E^{C}$, magnetic helicity $H^{M}$, and other anisotropies, would remain for finite $k$ due to the effect coming from the large scales. If there is a strong mean magnetic field at the large scales, the effect from it on the anisotropy in small scales is of particular interest. The divergence of the integral due to $Q^{B}$ in the analysis in Sec. IV suggests that the large scales of the magnetic field influence the statistics of the small scales more strongly than those of the velocity field. Hence, the universal spectrum in the inertial subrange of the MHD turbulence is possibly less robust than that of the purely hydrodynamic turbulence. In principle, the helical or anisotropic components of $Q_{i j}^{\alpha \beta}(\mathbf{k})$ can be analyzed by using the LRA equations (2.14)-(2.17). Pioneering analyses using LRA of anisotropic MHD turbulence under a strong uniform mean magnetic field were made by Nakayama (see Sec. I). For the purely hydrodynamic case, LRA was applied to the anisotropic spectrum of homogeneous turbulent shear flow in Ref. 26 , where the equations were simplified by taking into account the symmetry of the problem. By using a similar method, the LRA equations (2.14)-(2.17) for MHD turbulence may be simplified into forms that are convenient for the analyses of helical or anisotropic components of the spectrum tensor. Such analyses are left for future studies.

\section{ACKNOWLEDGMENTS}

The authors are grateful to Y. Kaneda, T. Gotoh, W. Bos and the anonymous referee for stimulating and useful discussions, and W.-C. Müller for showing data from his DNS. The DNSs in this research were performed on a Fujitsu HPC2500 system at the Information Technology Center, Nagoya University. This research is partially supported by Grant-in-Aid for Young Scientists (B) No. 17740246 from the Ministry of Education, Culture, Sports, Science, and Technology of Ja- 
pan, and by Grant-in-Aid for Scientific Research (B) No. 17340117 from the Japan Society for the Promotion of Science.

\section{APPENDIX: METHOD TO GENERATE THE RANDOM FORCES}

Let $W^{(i)}(\mathbf{k}, t)(i=1,2)$ be random variables that take values 1 or -1 with the equal probability and satisfy

$$
\left\langle W^{(i)}(\mathbf{k}, t)\right\rangle=0, \quad\left\langle W^{(i)}(\mathbf{k}, t) W^{(j)}\left(\mathbf{k}^{\prime}, t^{\prime}\right)\right\rangle=\delta_{\mathbf{k}, \mathbf{k}^{\prime}} \delta_{t, t^{\prime}} \delta_{i j} .
$$

Let $\Theta(\mathbf{k}, t)$ be a random variable that is uniformly distributed in $[-\pi, \pi)$ and satisfies

$$
\langle\Theta(\mathbf{k}, t)\rangle=0, \quad\left\langle\Theta(\mathbf{k}, t) \Theta\left(\mathbf{k}^{\prime}, t^{\prime}\right)\right\rangle=\frac{\pi^{2}}{3} \delta_{\mathbf{k}, \mathbf{k}^{\prime}} \delta_{t, t^{\prime}} .
$$

Provided that $\Delta t \ll T_{f}$, the random forces $\mathbf{f}^{u}(\mathbf{k}, t)$ and $\mathbf{f}^{B}(\mathbf{k}, t)$ with desired statistical properties (6.10)-(6.12) can be generated from $W^{(i)}(\mathbf{k}, t)$ and $\Theta(\mathbf{k}, t)$ by

$$
\begin{aligned}
& \mathbf{f}^{u}(\mathbf{k}, 0)=\sqrt{F^{u}} e^{i \Theta(\mathbf{k}, 0)} \frac{\mathbf{k}}{k} \times \mathbf{W}(\mathbf{k}, 0), \\
& \mathbf{f}^{B}(\mathbf{k}, 0)=\sqrt{F^{B}} \mathbf{W}(\mathbf{k}, 0), \\
& \mathbf{f}^{u}(\mathbf{k}, t+\Delta t)=\gamma \mathbf{f}^{u}(\mathbf{k}, t)+\zeta^{u} e^{i \Theta(\mathbf{k}, t)} \sqrt{\Delta t} \frac{\mathbf{k}}{k} \times \mathbf{W}(\mathbf{k}, t), \\
& \mathbf{f}^{B}(\mathbf{k}, t+\Delta t)=\gamma \mathbf{f}^{B}(\mathbf{k}, t)+\zeta^{B} \sqrt{\Delta t} \mathbf{W}(\mathbf{k}, t),
\end{aligned}
$$

for the cases (i) $k_{3}>0$, (ii) $k_{2}>0$, and $k_{3}=0$, and (iii) $k_{1}$ $>0, k_{2}=0$, and $k_{3}=0$, and

$$
\mathbf{f}^{u}(\mathbf{k}, t)=\mathbf{f}^{u^{*}}(-\mathbf{k}, t), \quad \mathbf{f}^{B}(\mathbf{k}, t)=\mathbf{f}^{B^{*}}(-\mathbf{k}, t)
$$

otherwise. Here,

$$
\begin{aligned}
& \gamma:=e^{-\frac{\Delta t}{T_{f}}}, \quad \zeta^{u}:=\sqrt{\frac{2 F^{u}}{T_{f}}}, \quad \zeta^{B}:=\sqrt{\frac{2 F^{B}}{T_{f}}}, \\
& \mathbf{e}^{(1)}(\mathbf{k}):=\frac{\mathbf{i}_{3} \times \mathbf{k}}{\left|\mathbf{i}_{3} \times \mathbf{k}\right|}, \quad \mathbf{e}^{(2)}(\mathbf{k}):=\frac{\mathbf{k} \times \mathbf{e}^{(1)}(\mathbf{k})}{\left|\mathbf{k} \times \mathbf{e}^{(1)}(\mathbf{k})\right|}, \\
& \mathbf{W}(\mathbf{k}, t):=W^{(1)}(\mathbf{k}, t) \mathbf{e}^{(1)}(\mathbf{k})+W^{(2)}(\mathbf{k}, t) \mathbf{e}^{(2)}(\mathbf{k}),
\end{aligned}
$$

and $\mathbf{i}_{3}$ is the unit vector along the $x_{3}$ axis.

${ }^{1}$ D. Biskamp, Magnetohydrodynamic Turbulence (Cambridge University Press, 2003).

${ }^{2}$ P. S. Iroshnikov, "Turbulence of a conducting fluid in a strong magnetic field," Sov. Astron. 7, 566 (1964)

${ }^{3}$ R. H. Kraichnan, "Inertial-range spectrum of hydromagnetic turbulence," Phys. Fluids 8, 1385 (1965).

${ }^{4}$ A. Pouquet, U. Frisch, and J. Léorat, "Strong MHD helical turbulence and the nonlinear dynamo effect," J. Fluid Mech. 77, 321 (1976).

${ }^{5}$ R. Grappin, U. Frisch, J. Léorat, and A. Pouquet, "Alfvenic fluctuations as asymptotic states of MHD turbulence," Astron. Astrophys. 105, 6 (1982).

${ }^{6} \mathrm{R}$. Grappin, A. Pouquet, and J. Léorat, "Dependence of MHD turbulence spectra on the velocity field-magnetic field correlation," Astron. Astrophys. 126, 51 (1983).

${ }^{7}$ S. Sridhar and P. Goldreich, "Toward a theory of interstellar turbulence. I. Weak Alfvénic turbulence," Astrophys. J. 432, 612 (1994).

${ }^{8}$ P. Goldreich and S. Sridhar, "Toward a theory of interstellar turbulence. II. Strong Alfvénic turbulence," Astrophys. J. 438, 763 (1995).

${ }^{9}$ P. Goldreich and S. Sridhar, "Magnetohydrodynamic turbulence revisited," Astrophys. J. 485, 680 (1997).

${ }^{10}$ W.-C. Müller and D. Biskamp, "The evolving phenomenological view on magnetohydrodynamic turbulence," in Turbulence and Magnetic Fields in Astrophysics, edited by E. Falgarone and T. Passot (Springer, Berlin, 2002), pp. 3-27.

${ }^{11}$ S. Galtier, S. V. Nazarenko, A. C. Newell, and A. Pouquet, "A weak turbulence theory for incompressible magnetohydrodynamics," J. Plasma Phys. 63, 447 (2000).

${ }^{12}$ D. Biskamp and W.-C. Müller, "Scaling properties of three-dimensional isotropic magnetohydrodynamic turbulence," Phys. Plasmas 7, 4889 (2000), referred to as BM.

${ }^{13}$ W.-C. Müller and R. Grappin, "Spectral energy dynamics in magnetohydrodynamic turbulence," Phys. Rev. Lett. 95, 114502 (2005), referred to as MG.

${ }^{14}$ Y. Kaneda, "Renormalized expansions in the theory of turbulence with the use of the Lagrangian position function,” J. Fluid Mech. 107, 131 (1981).

${ }^{15}$ Y. Kaneda and T. Gotoh, "General expression and applications of Lagrangian renormalized closure," in Research Report of Research Institute for Mathematical Science (Kyoto University, 1987), Vol. 652, pp. 159-171 (in Japanese).

${ }^{16}$ R. H. Kraichnan, "Lagrangian-history closure approximation for turbulence," Phys. Fluids 8, 575 (1965).

${ }^{17}$ R. H. Kraichnan and J. R. Herring, "A strain-based Lagrangian-history turbulence theory," J. Fluid Mech. 88, 355 (1978).

${ }^{18}$ V. I. Belinicher and V. S. L'vov, "A scale-invariant theory of fully developed hydrodynamic turbulence," Sov. Phys. JETP 66, 303 (1987).

${ }^{19}$ K. Nakayama, "Statistical theory of anisotropic magnetohydrodynamic turbulence. II. Lagrangian theory of strong shear Alfvén turbulence," Astrophys. J. 556, 1027 (2001).

${ }^{20} \mathrm{~K}$. Nakayama, "Lagrangian statistical theory of anisotropic MHD turbulence," Publ. Astron. Soc. Jpn. 54, 1065 (2002).

${ }^{21}$ R. H. Kraichnan, "Isotropic turbulence and inertial-range structure," Phys. Fluids 9, 1728 (1966).

${ }^{22}$ R. H. Kraichnan, "Eddy viscosity in two and three dimensions," J. Atmos. Sci. 33, 1521 (1976).

${ }^{23}$ D. C. Leslie, Developments in the Theory of Turbulence (Clarendon Press, Oxford, 1973), Chap. 6.

${ }^{24} \mathrm{Y}$. Kaneda, "Inertial range structure of turbulent velocity and scalar fields in a Lagrangian renormalized approximation," Phys. Fluids 29, 701 (1986).

${ }^{25}$ R. H. Kraichnan, "The structure of isotropic turbulence at very high Reynolds numbers," J. Fluid Mech. 5, 497 (1959).

${ }^{26} \mathrm{~K}$. Yoshida, T. Ishihara, and Y. Kaneda, "Anisotropic spectrum of homogeneous turbulent shear flow in a Lagrangian renormalized approximation," Phys. Fluids 15, 2385 (2003).

${ }^{27}$ Y. Kaneda and K. Yoshida, "Small-scale anisotropy in stably stratified turbulence," New J. Phys. 6, 34 (2004).

${ }^{28}$ Y. Kaneda, T. Ishihara, M. Yokokawa, K. Itakura, and A. Uno, "Energy dissipation rate and energy spectrum in high resolution direct numerical simulations of turbulence in a periodic box," Phys. Fluids 15, L21 (2003).

${ }^{29}$ N. E. L. Haugen, A. Brandenburg, and W. Dobler, "Simulation of nonhelical hydromagnetic turbulence," Phys. Rev. E 70, 016308 (2004).

${ }^{30}$ M. L. Goldstein and D. A. Roberts, "Magnetohydrodynamic turbulence in the solar wind," Phys. Plasmas 6, 4154 (1999). 